\title{
Human autologous iPSC-derived dopaminergic progenitors restore motor function in Parkinson's disease models
}

\author{
Bin Song, ${ }^{1,2}$ Young Cha, ${ }^{1,2}$ Sanghyeok Ko, ${ }^{1,2}$ Jeha Jeon, ${ }^{1,2}$ Nayeon Lee, ${ }^{1,2}$ Hyemyung Seo, ${ }^{1,2,3}$ Kyung-Joon Park, ${ }^{1}$ In-Hee Lee, ${ }^{4,5}$ \\ Claudia Lopes, ${ }^{1,2}$ Melissa Feitosa, ${ }^{1,2}$ María José Luna, ${ }^{1,2}$ Jin Hyuk Jung, ${ }^{1,2}$ Jisun Kim, ${ }^{1,2,3}$ Dabin Hwang, ${ }^{1,2}$ Bruce M. Cohen, ${ }^{1}$ \\ Martin H. Teicher, ${ }^{1}$ Pierre Leblanc, ${ }^{1,2}$ Bob S. Carter, ${ }^{6}$ Jeffrey H. Kordower, ${ }^{7}$ Vadim Y. Bolshakov, ${ }^{1}$ Sek Won Kong, ${ }^{4,5}$ \\ Jeffrey S. Schweitzer, ${ }^{6}$ and Kwang-Soo Kim ${ }^{1,2}$ \\ 'Department of Psychiatry and ${ }^{1}$ Molecular Neurobiology Laboratory, McLean Hospital, Harvard Medical School, Belmont, Massachusetts, USA. ${ }^{3}$ Department of Molecular and Life Sciences, \\ Hanyang University, Ansan, Korea. ${ }^{4}$ Department of Pediatrics, ${ }^{5}$ Computational Health Informatics Program, Boston Children's Hospital, and ${ }^{6}$ Department of Neurosurgery, Massachusetts General Hospital, \\ Harvard Medical School, Boston, Massachusetts, USA. D.pepartment of Neurological Sciences, Rush University Medical Center, Chicago, Illinois, USA.
}

\begin{abstract}
Parkinson's disease (PD) is a neurodegenerative disorder associated with loss of striatal dopamine, secondary to degeneration of midbrain dopamine ( $\mathrm{mDA}$ ) neurons in the substantia nigra, rendering cell transplantation a promising therapeutic strategy. To establish human induced pluripotent stem cell-based (hiPSC-based) autologous cell therapy, we report a platform of core techniques for the production of mDA progenitors as a safe and effective therapeutic product. First, by combining metabolism-regulating microRNAs with reprogramming factors, we developed a method to more efficiently generate clinical-grade iPSCs, as evidenced by genomic integrity and unbiased pluripotent potential. Second, we established a "spotting"-based in vitro differentiation methodology to generate functional and healthy mDA cells in a scalable manner. Third, we developed a chemical method that safely eliminates undifferentiated cells from the final product. Dopaminergic cells thus express high levels of characteristic mDA markers, produce and secrete dopamine, and exhibit electrophysiological features typical of mDA cells. Transplantation of these cells into rodent models of PD robustly restores motor function and reinnervates host brain, while showing no evidence of tumor formation or redistribution of the implanted cells. We propose that this platform is suitable for the successful implementation of human personalized autologous cell therapy for PD.
\end{abstract}

\section{Introduction}

Parkinson's disease (PD), characterized by both motor and nonmotor system pathology, is a common neurodegenerative disorder affecting about $1 \%$ of the population over age 60 . Its prevalence presents an increasing social burden as the population ages (1). Since its introduction in the 1960s, dopamine-replacement (DA-replacement) therapy (e.g., L-DOPA) has remained the gold standard treatment. While this treatment improves PD patients' quality of life, the effects fade with disease progression and prolonged usage of these medications often $(>80 \%)$ results in side effects, including dyskinesias and motor fluctuations (2). Since the selective degeneration of $\mathrm{A} 9$ midbrain $\mathrm{DA}$ (mDA) neurons (mDANs) in the substantia nigra (SN) is a key pathological feature of the disease and is directly associated with the cardinal motor

\section{Related Commentary: p. 601}

Authorship note: BS, YC, SK, JJ, NL, and HS contributed equally to this work. Conflict of interest: The authors have declared that no conflict of interest exists. Copyright: (5) 2020, American Society for Clinical Investigation.

Submitted: June 3, 2019; Accepted: October 30, 2019; Published: January 13, 2020

Reference information: / Clin Invest. 2020;130(2):904-920.

https://doi.org/10.1172/JCl130767. symptoms, dopaminergic cell transplantation has been proposed as a therapeutic strategy (3). In support of this, previous fetal cell transplantation provided proof of concept in which grafts successfully reinnervated target areas with varying degrees of restoration of function, in some patients providing substantial recovery lasting decades (4-7). Despite these promising results, tissue derived from human fetuses has fundamental ethical, practical, and medical limitations as a cell source for treatment of PD.

In 2006, Yamanaka and colleagues published a groundbreaking study showing that mammalian fibroblasts can be converted into embryonic stem cell-like (ESC-like) induced pluripotent stem cells (iPSCs) by introducing 4 transcription factors, i.e., Oct4, Sox2, Klf4, and c-Myc (hereafter denoted as Y4F [Yamanaka 4 factors]) (8). Yamanaka's and 2 other groups subsequently accomplished this feat with human somatic cells, reprograming them into human iPSCs (hiPSCs) (9-11), offering the possibility of generating patient-specific stem cells. Despite initial excitement, it remains uncertain whether this hiPSC technology can readily be used for autologous cell therapy. Indeed, the major goals of most hiPSC research have moved from personalized cell therapy toward mechanistic studies of human disease and development (12). There are several major barriers to implementation of hiPSC-based cell therapy for PD. First, probably due to our limited 
A
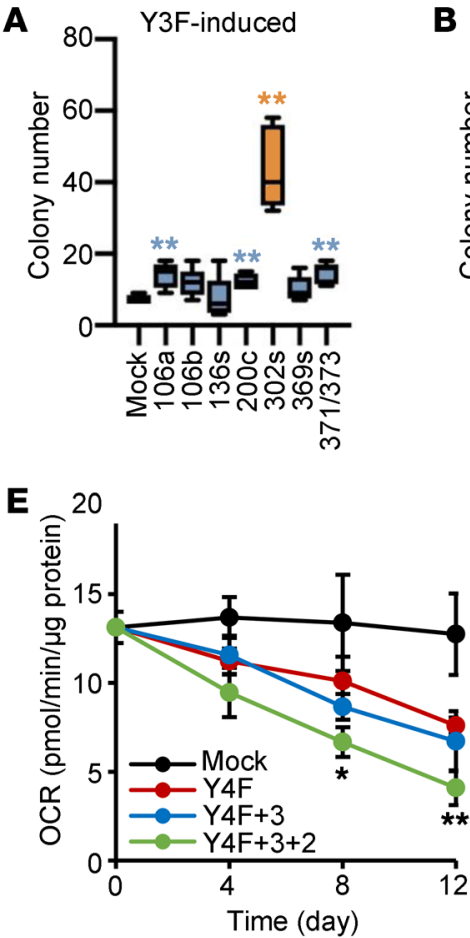

B

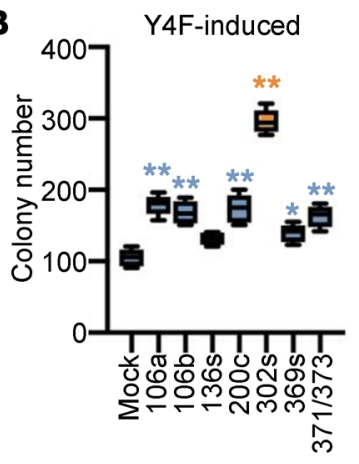

$\mathbf{F}$

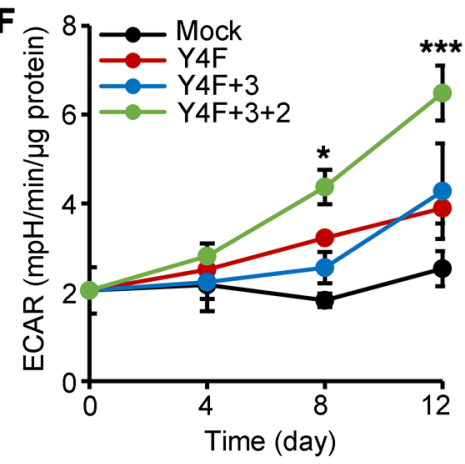

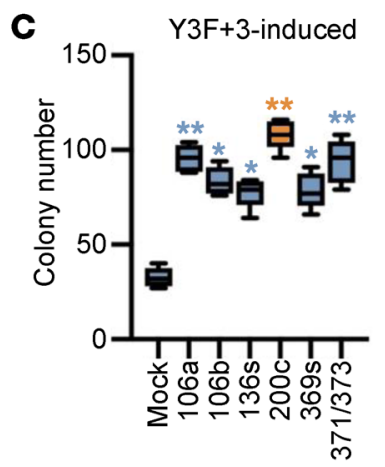

G

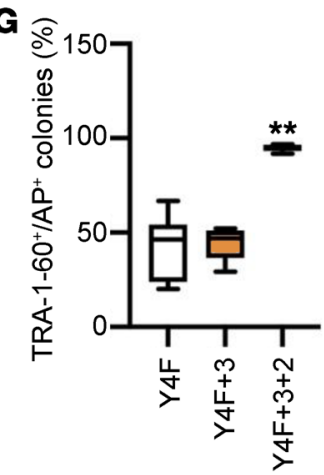

D

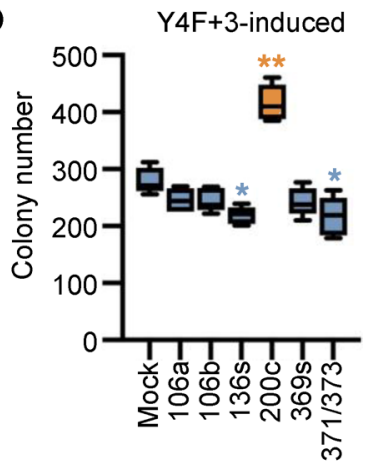

$\mathbf{H}$

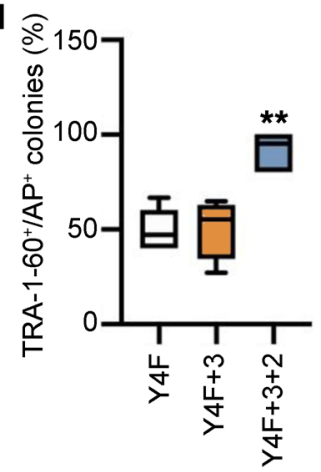

Figure 1. An improved reprogramming method combining Y4F and metabolism-regulating miRNAs. (A-D) Screening of miRNAs that enhance the generation of hiPSC-like colonies by $\mathrm{Y3F}(\mathbf{A}), \mathrm{Y} 4 \mathrm{~F}(\mathbf{B}), \mathrm{Y3F}+3$ (C), or $\mathrm{Y} 4 \mathrm{~F}+3$ (D) from hDFs, relative to an empty vector (mock) control. Mean \pm SD. $n=5$. ${ }^{*} P<0.05$ ${ }^{*} P<0.01$, 1-way ANOVA with Tukey's post test. (E and F) Time course of OCR (E) and ECAR (F) in hDFs infected with Y4F, miR-302s, and/or miR-200c. Mean \pm SD. $n=3$. ${ }^{*} P<0.05 ;{ }^{*} P<0.01 ;{ }^{* *} P<0.005,2$-way ANOVA with Tukey's post test. (G) Percentage of TRA-1-60 ${ }^{+}$colonies among AP ${ }^{+}$colonies following lentiviral infection encoding Y4F, Y4F+3, or Y4F+3+2. Mean \pm SD. $n=6$. ${ }^{* * *} P<0.005$, 2-way ANOVA with Tukey's post test. (H) Percentage of TRA-1-60+ colonies among $\mathrm{AP}^{+}$colonies following transfection with episomal vectors encoding $\mathrm{Y} 4 \mathrm{~F}, \mathrm{Y} 4 \mathrm{~F}+3$, or $\mathrm{Y} 4 \mathrm{~F}+3+2 . \mathrm{Mean} \pm \mathrm{SD}$. $n=4$. ${ }^{* *} P<0.01,2-\mathrm{way}^{*}$ ANOVA with Tukey's post test.

understanding of the reprogramming process, wide variability exists among the differentiation potentials of individual hiPSC lines $(13,14)$. Second, the safety of hiPSC-based cell therapy has yet to be fully established. In particular, since any hiPSCs that remain undifferentiated or bear subclonal tumorigenic mutations have neoplastic potential $(15,16)$, it is critical to eliminate completely such cells from a therapeutic product. As illustrated by 1 of 2 patients in the first hiPSC-based human trial (17), safe clinical use requires that genomic integrity of hiPSCs be confirmed by whole-genome sequencing/whole-exome sequencing (WGS/ WES). Third, despite numerous studies by multiple laboratories, in vitro differentiation protocols of hiPSCs into functional mDANs remain suboptimal, adding to end product variability $(7,18)$. Finally, long-term cost efficacy and reproducibility will be necessary to benefit as many patients as possible.

In this study, we sought to address these challenges. We propose that the core techniques described here offer a protocol suitable for the successful implementation of personalized, autologous cell-replacement therapy for PD.

\section{Results}

Identification of microRNAs that regulate metabolic reprogramming. We recently showed that SIRT2, directly targeted by miR-200c, is critical for metabolic reprogramming and hiPSC generation (19). To validate a functional link between miR-200c and repro- gramming, we tested to determine whether forced expression of miR-200c would induce a metabolic change. Indeed, miR-200c overexpression (OE) in human dermal fibroblasts (hDFs) resulted in significant metabolic changes, including decreased oxygen consumption rate (OCR) and increased extracellular acidification rate (ECAR) (Supplemental Figure 1, A and B; supplemental material available online with this article; https://doi.org/10.1172/ JCI130767DS1). Compared with empty-vector control lines, miR200c OE cells showed significantly decreased oxidative phosphorylation (OXPHOS) capacity, with decreases in basal respiration, ATP turnover, maximum respiration, and oxidative reserve, as well as OCR changes after carbonyl cyanide-p-trifluoromethoxyphenylhydrazone (FCCP) injection (Supplemental Figure 1, C-E). We next treated hDFs with reprogramming factors (i.e., Y4F) together with miR-200c. Addition of miR-200c OE significantly reduced OXPHOS, compared with Y4F only (Supplemental Figure 1, F-K), suggesting that pluripotency-associated microRNAs (miRNAs) influence the reprogramming process by facilitating metabolic reprogramming. To test this, we investigated whether other miRNAs could, like miR-200c, induce metabolic changes. Based on previous miRNA expression studies (20-23), we identified 8 candidate miRNA clusters (miR-17/92, miR-106a, miR-106b, miR-136s, miR-200c, miR-302s, miR-369s, and miR371/373) that are consistently enriched in hPSCs. We tested to determine whether OE of these miRNAs in hDFs would lead to 
A

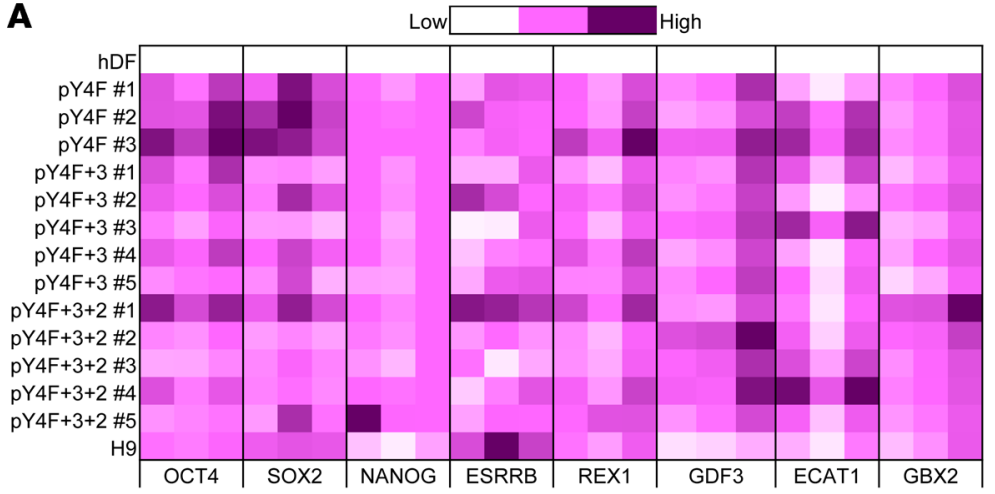

B

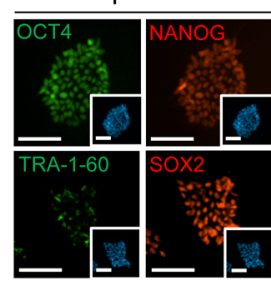

$\mathrm{pY} 4 \mathrm{~F}+3$

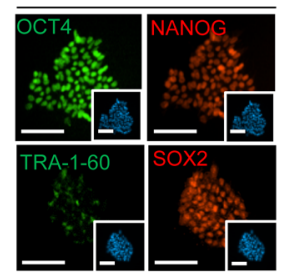

pY $4 \mathrm{~F}+3+2$

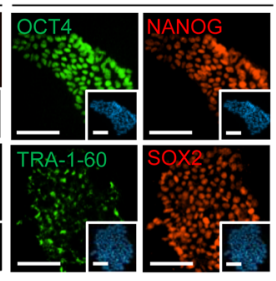

H9

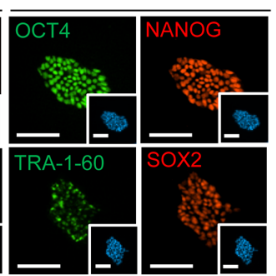

C

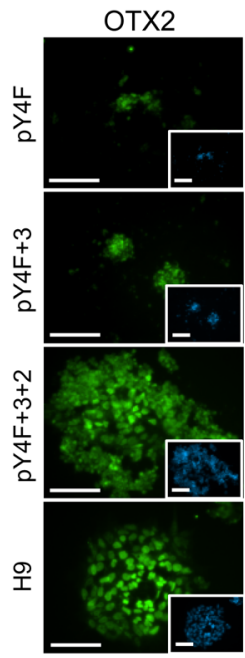

B-T

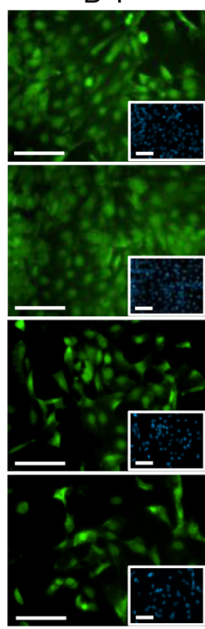

SOX17

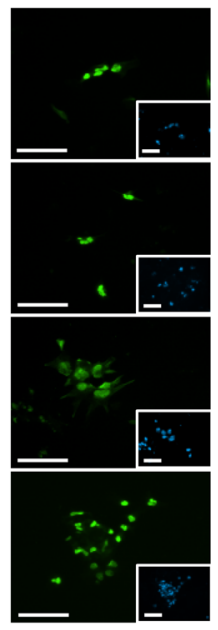

D

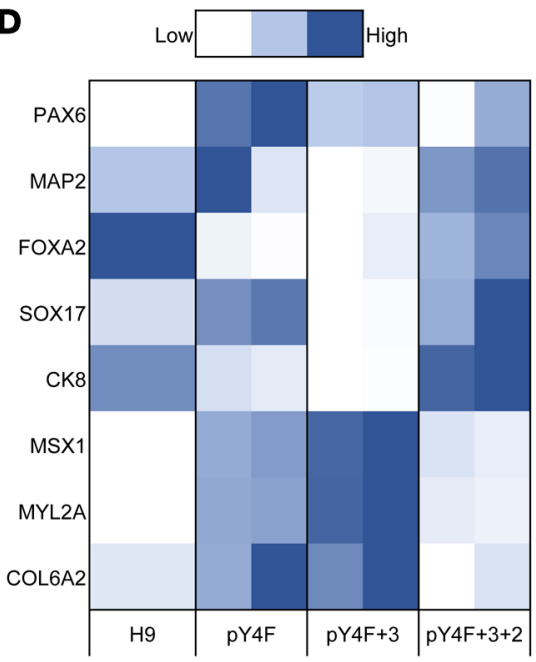

Figure 2. Higher quality hiPSC lines generated from our improved reprogramming method. (A) Heatmaps depicting gene expression levels of pluripotency markers among established hiPSC lines compared with the original hDFs and an hESC line (H9). $n=3$. (B) Immunostaining of hiPSC lines generated by different combinations with specific antibodies against pluripotency markers (e.g., OCT4, NANOG, TRA-1-60, and SOX2) along with Hoechst 33342 nuclear staining (insets). Scale bars: $100 \mu \mathrm{m}$. (C) Immunostaining for lineage-specific markers for ectoderm (OTX2), mesoderm (BRACHYURY), and endoderm (SOX17) following spontaneous differentiation for 7 days. Scale bars: $100 \mu \mathrm{m}$. (D) Heatmaps depicting gene expression levels of early differentiation markers of ectoderm (PAX6 and MAP2), endoderm (FOXA2, SOX17, and CK8), and mesoderm markers (MSX1, MYL2A, and COL6A2) in hiPSC lines generated by $\mathrm{pY} 4 \mathrm{~F}, \mathrm{pY} 4 \mathrm{~F}+3$, or $\mathrm{pY} 4 \mathrm{~F}+3+2 . n=2$.

metabolic changes. Interestingly, we found that 7 out of these 8 miRNA clusters (excepting miR-17/92) resulted in significant metabolic reprogramming, including decreased OCR and increased ECAR, leading to robust reductions in the OCR/ECAR ratio ranging from 1:3 to 1:20 compared with control cells transduced with empty vector (Supplemental Figure 1, L-N).

Combining metabolism-regulating miRNAs with reprogramming factors efficiently generates high-quality hiPSCs. We tested to determine whether adding these metabolism-regulating miRNAs to the usual reprogramming factors (either Y4F or Y3F [OCT4, SOX2, and KLF4]) on lentiviral vectors would facilitate generation of hiPSCs. Among the 7 miRNA clusters identified above, miR-302s exhibited the highest potency in enhancing hiPSC generation when combined with Y3F or Y4F (Figure 1, A and B). In addition, miR-106a, miR-106b, miR-200c, miR-369s, or miR-371/373 clusters also modestly but substantially increased hiPSC genera- tion. We next tested to determine whether any additional miRNA would further enhance hiPSC generation in combination with $\mathrm{Y} 3 \mathrm{~F}$ and miR-302s $(\mathrm{Y} 3 \mathrm{~F}+3)$ or with $\mathrm{Y} 4 \mathrm{~F}$ and $\mathrm{miR}-302 \mathrm{~s}(\mathrm{Y} 4 \mathrm{~F}+3)$. In the presence of $\mathrm{Y} 3 \mathrm{~F}+3$, addition of any of the other miRNA clusters did significantly enhance hiPSC generation (Figure 1C). When $\mathrm{Y} 4 \mathrm{~F}+3$ was used, only miR-200c significantly enhanced hiPSC generation (Figure 1D), thus identifying the combination of $\mathrm{Y} 4 \mathrm{~F}$, miR-302s, and miR-200c ( $\mathrm{Y} 4 \mathrm{~F}+3+2)$ as optimal. We compared the dynamics of metabolic changes during reprogramming induced by $\mathrm{Y} 4 \mathrm{~F}, \mathrm{Y} 4 \mathrm{~F}+3$, and $\mathrm{Y} 4 \mathrm{~F}+3+2$. Notably, $\mathrm{Y} 4 \mathrm{~F}+3+2$ induced the most prominent metabolic change (Figure 1, E and F), supporting a link between metabolic change and efficient hiPSC generation. We next investigated whether this combination could also influence the overall quality of hiPSCs by staining for alkaline phosphatase (AP) and for a more stringent pluripotency marker, TRA-1-60 $(24,25)$. Approximately $40 \%$ of $\mathrm{AP}^{+}$colonies generated by $\mathrm{Y} 4 \mathrm{~F}$ 
A
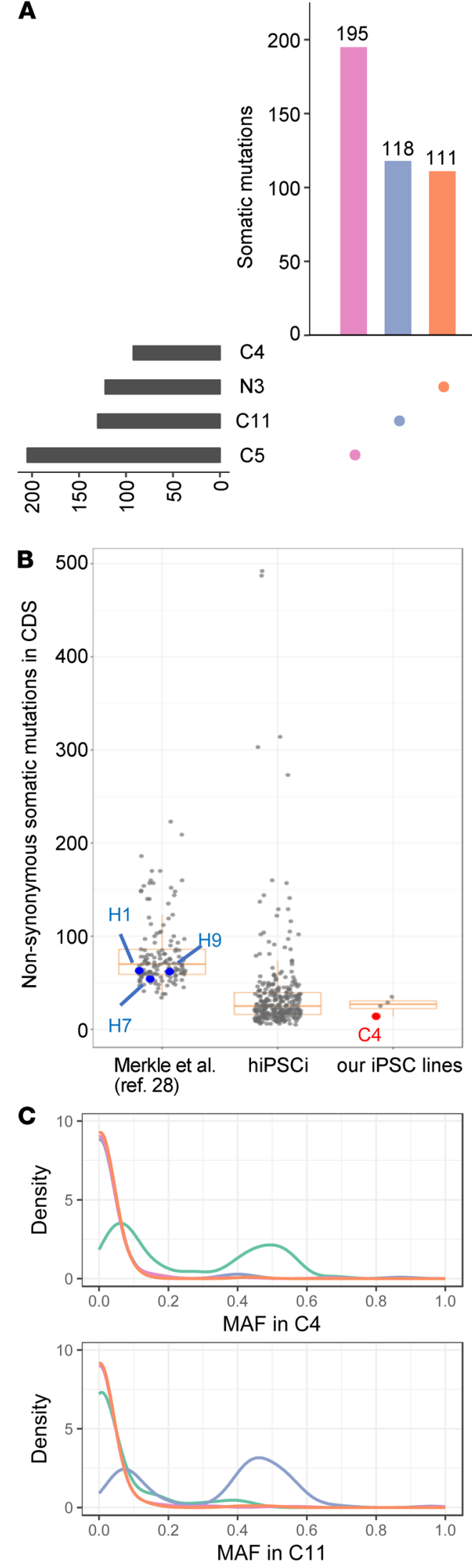
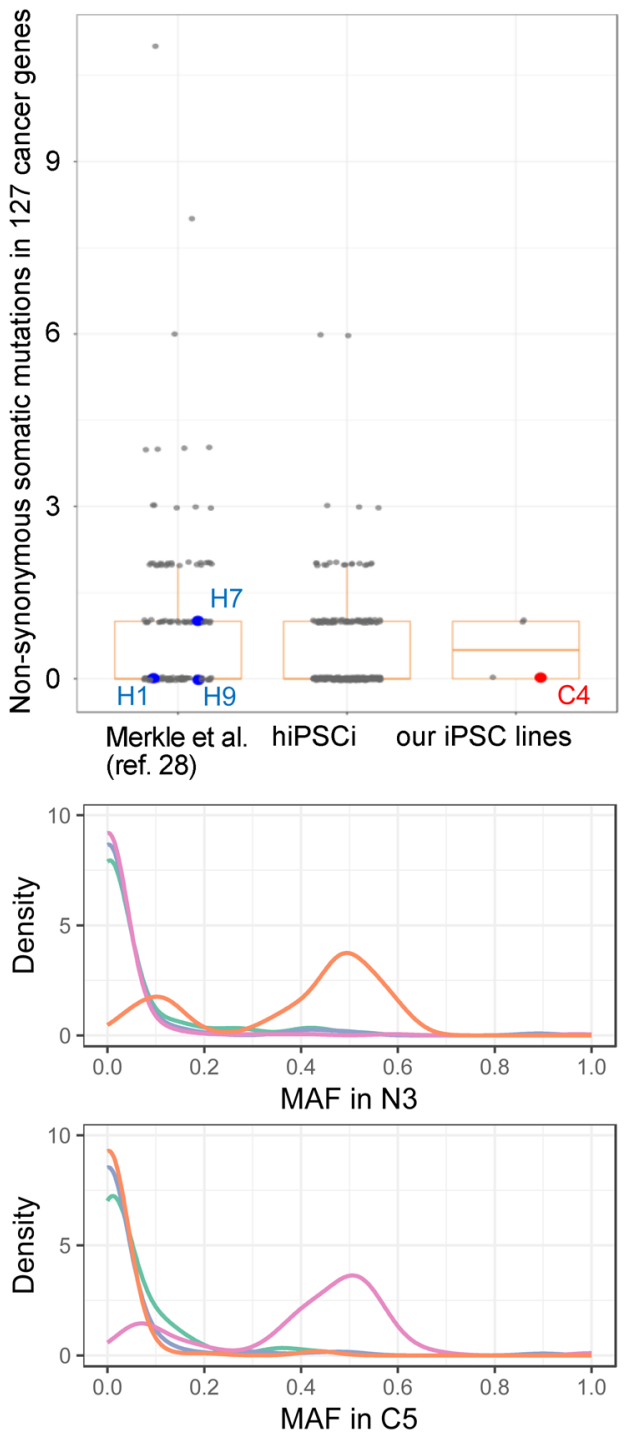

N3 C11

C5
Figure 3. Genomic integrity of hiPSC lines generated from skin biopsy of a sporadic PD patient. (A) Somatic mutations found in 4 hiPSC lines. Columns show the number of singleton mutations in each hiPSC line (different colors per hiPSC line) and number of unique mutations found in 2 or more hiPSC lines (black columns). Below black columns, hiPSC lines sharing the mutations are indicated by dots connected with edges. Bottom left bars represent total numbers of mutations including both singleton and those found in 2 or more hiPSC lines. C4 had the smallest number of somatic mutations ( $n$ = 92), of which 80 were singleton and 12 were found in $\mathrm{C} 4$ and the other hiPSC lines. (B) Mutational burdens on coding regions and cancer-associated genes were compared with publicly available data sets. The numbers of nonsynonymous mutations in our hiPSC lines were significantly lower than for hESC lines. On average, the numbers of nonsynonymous mutations in the IPSC lines from the HipSci project (28) are similar to those of our hiPSC lines. Overall, C4 shows the lowest mutation burden (red). For the somatic mutations in cancer-associated genes, no somatic mutation was found in 2 widely used hESC lines ( $\mathrm{H} 1$ and $\mathrm{H} 9$, blue) and C4 hiPSC line (red) (right panel). (C) Distribution of minor allelic fractions (MAFs) of all somatic mutations in the 4 hiPSC lines. The peaks around MAF of 0.5 denote clonal somatic mutations. The second peaks with lower MAFs of 0.1 denote subclonal mutations. For each plot, the density curve with 2 peaks shows the distribution of somatic mutation MAFs. The colors of curves match with those in $\mathbf{A}$ for each hiPSC line. Curves with different colors (peaked around MAF of 0.0 ) indicate somatic mutations detected by the other hiPSC lines. 
or $\mathrm{Y} 4 \mathrm{~F}+3$ were $\mathrm{TRA}-1-60^{+}$. In contrast, over $90 \%$ of $\mathrm{AP}^{+}$colonies generated by $\mathrm{Y} 4 \mathrm{~F}+3+2$ were TRA-1-60+ ${ }^{+}$(Figure $1 \mathrm{G}$ and Supplemental Figure 2A). Moreover, TRA-1-60+ hiPSC colonies generated by $\mathrm{Y} 4+3+2$ showed typical hESC-like compact colony morphology (Supplemental Figure 2A). We also reprogrammed adult hDFs (GM03529, Coriell Institute) and found that over $90 \%$ of colonies generated by $\mathrm{Y} 4 \mathrm{~F}+3+2$ on lentiviral vectors were $\mathrm{AP}^{+} \mathrm{TRA}-1-60^{+}$ (Supplemental Figure 2B).

We next tested to determine whether this combination $(\mathrm{Y} 4 \mathrm{~F}+3+2)$ could generate high-quality hiPSCs using nonviral vectors. We developed 2 episomal vectors harboring $\mathrm{Y} 4 \mathrm{~F}$ on 1 vector (pY4F; Supplemental Figure 2C) and miR-302s and miR-200c clusters on the other $(\mathrm{p} 3+2$; Supplemental Figure 2D). Because of the known transformation activity of c-Myc (26), we replaced it with L-MYC on pY4F. We thus established an episomal reprogramming protocol using single transfection with these 2 vectors (Supplemental Figure 2E) that efficiently reprogrammed hDFs to hiPSC colonies that were more than 90\% $\mathrm{AP}^{+} \mathrm{TRA}^{-1}-60^{+}$(Figure 1H). We selected hiPSC lines with hESC-like morphology generated by $\mathrm{Y} 4 \mathrm{~F}, \mathrm{Y} 4 \mathrm{~F}+3$, and $\mathrm{Y} 4 \mathrm{~F}+3+2$, passaged them more than 20 times, and characterized their properties. As shown in Figure 2, A and B, their morphologies and expression levels of pluripotency markers closely resembled those of $\mathrm{H} 9 \mathrm{hESC}$. Interestingly, H9 and hiPSCs generated by $\mathrm{Y} 4 \mathrm{~F}+3+2$ differentiated equally well to all 3 germ layer lineages, while differentiation of those generated by $\mathrm{Y} 4 \mathrm{~F}$ or $\mathrm{Y} 4 \mathrm{~F}+3$ was skewed toward mesodermal lineage, as evidenced by (a) staining with antibodies against the 3 germ layer markers and (b) gene expression of lineage-specific markers (Figure 2, C and D). These results suggest that the $\mathrm{Y} 4 \mathrm{~F}+3+2$ combination enables the generation of higher quality hiPSCs from both newborn and adult human fibroblasts with less biased differentiation potential, regardless of the delivery vector, compared with conventional methods (Y4F or $\mathrm{Y} 4 \mathrm{~F}+3$ ) (Supplemental Table 1).

Genomic integrity and somatic mutations in hiPSCs. To determine whether our reprogramming method can reliably generate clinical grade hiPSCs, we attempted to generate hiPSC lines using adult hDFs from multiple sources, including 9 fibroblast lines from the Coriell Institute ( 3 familial PD, 3 sporadic PD, and 3 healthy subjects) and 4 samples from new skin biopsies ( 3 healthy subjects and 1 sporadic PD patient). As shown in Supplemental Table 2 and Supplemental Figure 3, A and B, our method generated multiple hiPSC lines from all of these fibroblasts using a 1-time transfection with pY4F and p3+2 (Supplemental Figure 2E), all displaying hESC-like morphology and prominent expression of pluripotent markers, including OCT4, TRA-1-60, NANOG, and SSEA-4.

Focusing on personalized cell therapy, we further characterized hiPSC clones made from skin biopsy of a sporadic PD patient (MCL540 in Supplemental Table 2). A fundamental criterion for clinical grade hiPSCs is maintenance of genomic integrity and absence of harmful (e.g., reported cancer causing) mutation(s) $(7,17)$. As an example, we tested 5 independent hiPSC clones that were passaged approximately 20 times since the original isolation from MCL540 (N17, C4, N3, C11, and C5) as well as control cells (parental fibroblasts and H9) for potential integration of vector DNAs into the host genome (Supplemental Table 3). To detect plasmid-derived sequences, we designed 8 sets of EBNA-1-spe- cific primers and identified 2 sets (EB-01 and EB-02) that specifically detect plasmid DNAs (Supplemental Figure 4A and data not shown). While plasmid DNAs were undetectable in cytoplasmic fractions (Supplemental Figure 4B), 1 (N17) of 5 clones showed an integrated plasmid sequence (Supplemental Figure 4C). Quantitative reverse-transcription PCR (qRT-PCR) analysis showed that N17 contained 1.3 to $1.7 \times 10^{4}$ copies of integrated plasmid sequences per 100 nanograms of genomic DNA (Supplemental Figure 4D). Since the amount of DNA in a diploid cell is about 6 picograms (http://bionumbers.hms.harvard.edu/bionumber. aspx?id $=111206$ ), 100 nanograms of genomic DNA used in qRTPCR represents about $1.76 \times 10^{4}$ cells. Thus, clone N17 appears to contain approximately 1 copy of plasmid sequences per cell. In contrast, 4 other clones and negative controls (original fibroblasts and H9) were free of integrated plasmid DNAs (Supplemental Figure 4D). We thus excluded N17 and further analyzed the remaining 4 hiPSC clones (C4, N3, C11, and $\mathrm{C} 5)$ by DNA fingerprinting, karyotyping, and in vivo pluripotent differentiation (Supplemental Figure 4, E-G).

We performed WES on these 4 hiPSC clones and found a total of 524 somatic mutations compared with the parent fibroblast DNA sequence, including 137 mutations in coding exons or in \pm 2 bps splicing acceptor and donor sites (Supplemental Table 4). Each hiPSC line carried a median of 126 somatic mutations (range 92-205), including a median of 114.5 singleton mutations (range 80-195). There were a few shared mutations $(n=1-4)$ between hiPSC lines (Figure 3A). C5 had the largest number of somatic mutations $(n=$ 205), and C4 had the fewest $(n=92)$, including 80 singletons. Of somatic mutations in protein-coding regions, hiPSC lines carried a median of 36.5 (range 17-50), including 27 (median, range 14-35) nonsynonymous mutations. Again, $\mathrm{C} 4$ had the fewest mutations. We investigated mutations in 127 genes reported as frequently mutated across multiple cancer types (27). Our hiPSC lines carried at most 1 mutation (synonymous or nonsynonymous) in these genes, and no nonsynonymous mutation was found from C4 or N3. In summary, of somatic mutations discovered in all 4 hiPSC lines, there were none causally implicated in cancer. Finally, we compared somatic mutation burden in our hiPSC lines with publicly available data sets (Figure 3B). We collected high-confidence somatic mutations from WES based on 140 hESC lines (28) and somatic coding mutations from WGS data for 299 hiPSC lines (generated by Sendai virus method) in the Human Induced Pluripotent Stem Cells Initiative (HipSci) (29). Our hiPSC lines showed overall mutational burdens similar to those of HipSci hiPSC lines (median 25, range 5-492) and significantly less than those of hESC lines (median 70, range 34-223) (Wilcoxon's rank sum test, $P$ value $=0.00071$ ) (Figure $3 \mathrm{~B})$. Also, our hiPSC lines carried smaller numbers of mutations in genes frequently mutated in cancer (Figure 3B).

We also checked for somatic mutations that might be present in a subpopulation in each hiPSC line. We estimated the distribution of allelic fractions in observed somatic mutations and performed binomial tests with a null model of $45 \%$ as the center for single nucleotide variants (SNVs) and $35 \%$ as the center for indels $(28,30)$. For each hiPSC line, a median of 16 (9-18 in range) variants, with Bonferroni's correction at $P<0.01$, were considered as potential candidates for somatic mutations originating from a fraction of cells (marked as subclonal in Supplemental Table 4). 
A

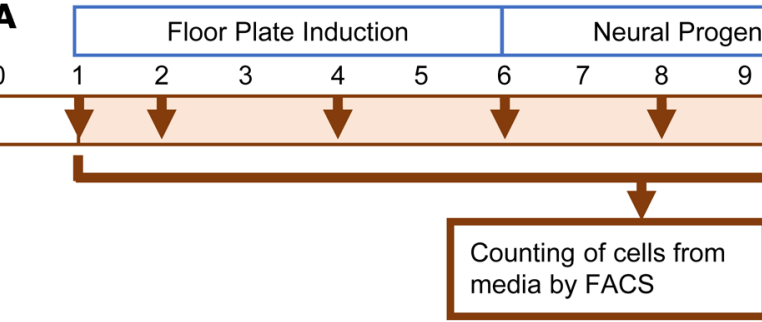

B

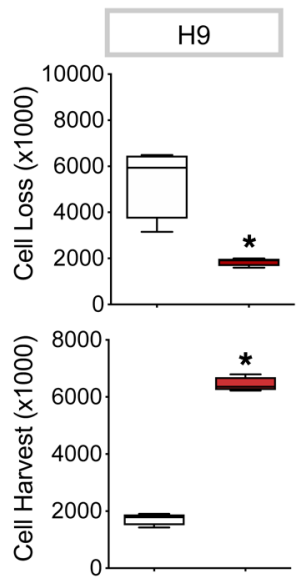

C
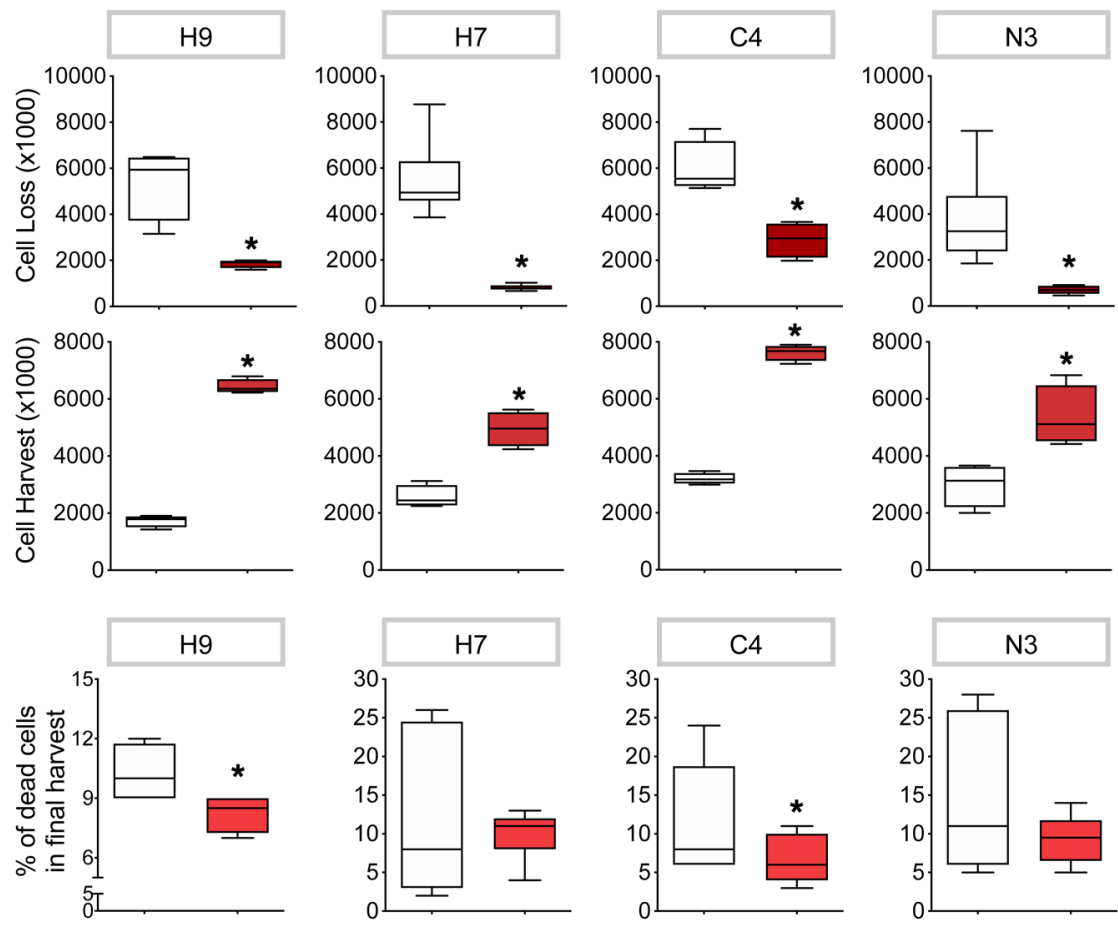

Cell Harvest \&

Characterization
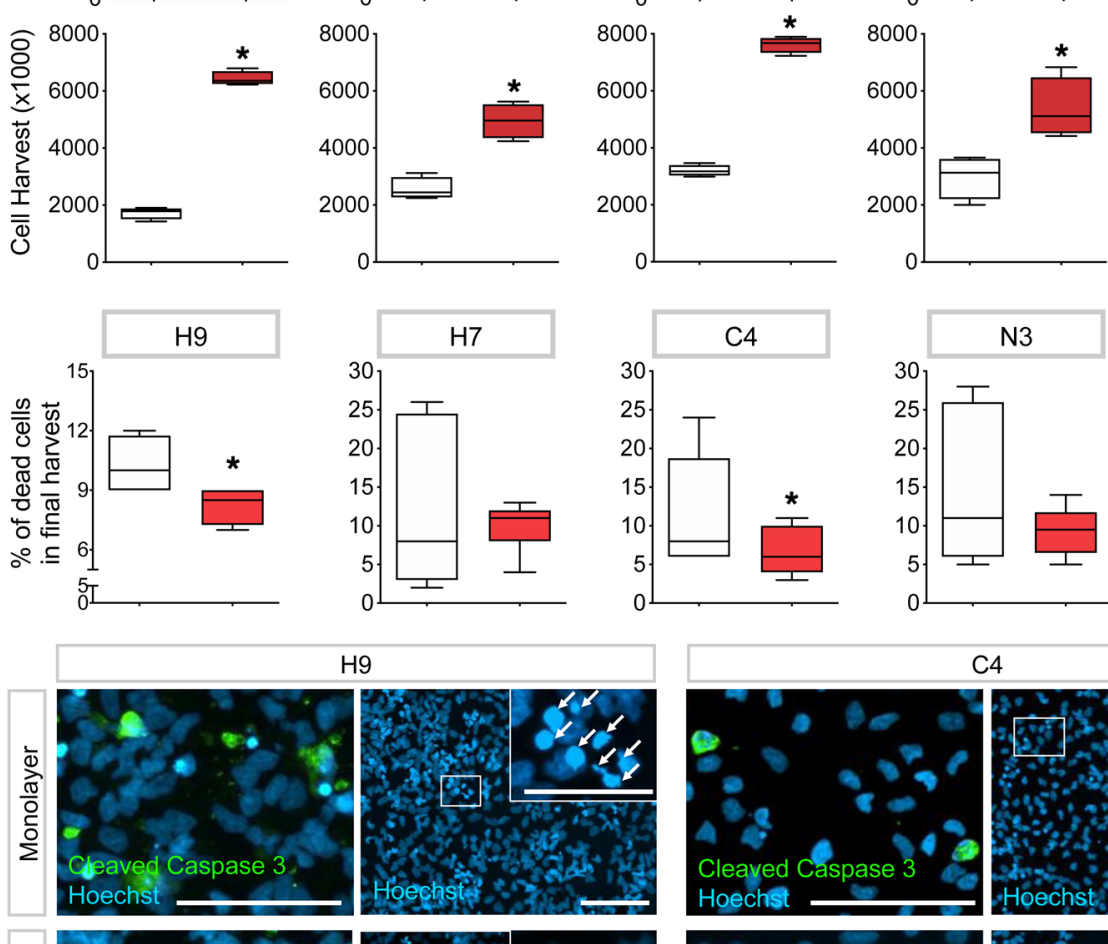

$\mathrm{H} 9$
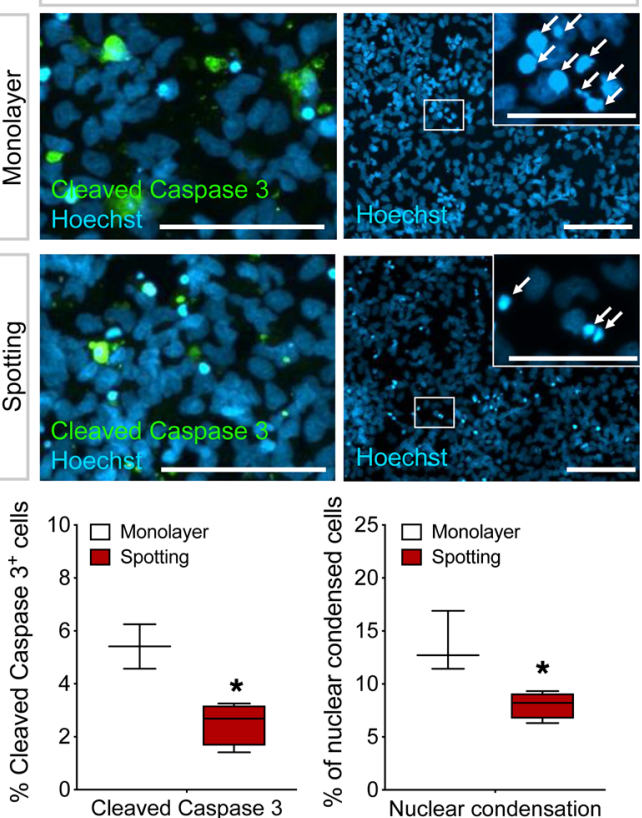

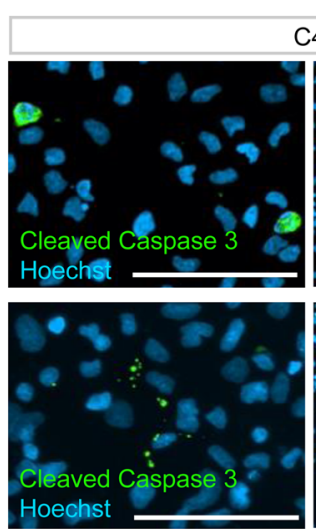

C4

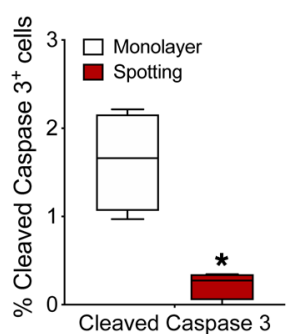

Monolayer

Spotting

Monolayer

Spotting

Monolayer Spotting

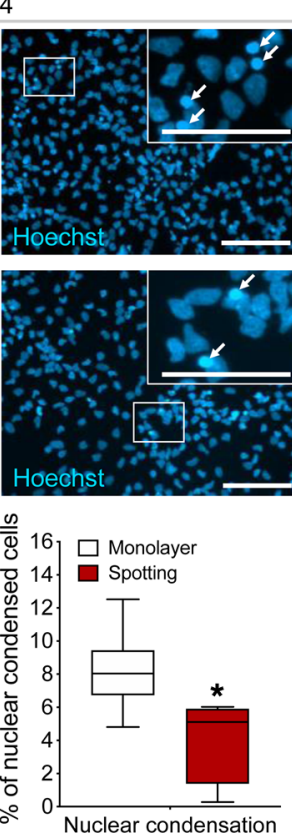

Figure 4. Spotting-based in vitro differentiation improves yield and quality of the resulting DA cells. (A) Experimental schematic to find optimized physical culture conditions. On d0 through d15, all cells regardless of viability were quantified by FACS and/or manual cell counting, as marked with arrows. At d15, cells were replated on cover glass for immunocytochemical analysis or harvested for qRT-PCR. (B and $\mathbf{C})$ Comparison between conventional monolayer-based and spotting-based methods for degree of cell loss (due to detachment) from d1 to d14 and cell harvest on $\mathrm{d} 15$ (B) and for the percentage of dead cells at d15 (C) for both hESC (H9 and $\mathrm{H} 7)$ and hiPSC (C4 and N3). Cell densities of $11,000 / \mathrm{cm}^{2}$ and 10,000 /spot were used for conventional and spotting-based methods, respectively. Data presented reflect experiments with measurable outcomes (see the legend of Supplemental Figure 5A). Mean \pm SD. $n=4$. One-way ANOVA. (D) Quantification of dying cells from final cell harvest on d15 using immunocytochemical analysis. Antibody against cleaved caspase-3 was used to detect apoptotic cells. Nuclear condensation was visualized by Hoechst 33342 staining to detect dead or dying cells. Cells plated with spotting technique showed significantly reduced numbers of cleaved caspase-3-positive cells. Scale bars: $100 \mu \mathrm{m}$. Scale bar (insets): $50 \mu \mathrm{m}$. 
A

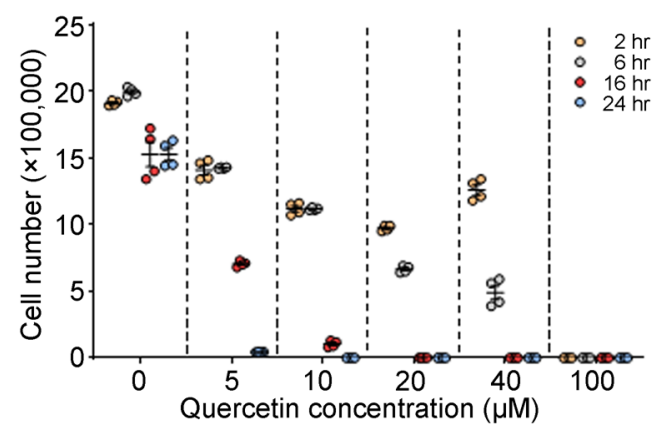

D hiPSCs input cell numbers per $10^{5}$ fibroblasts

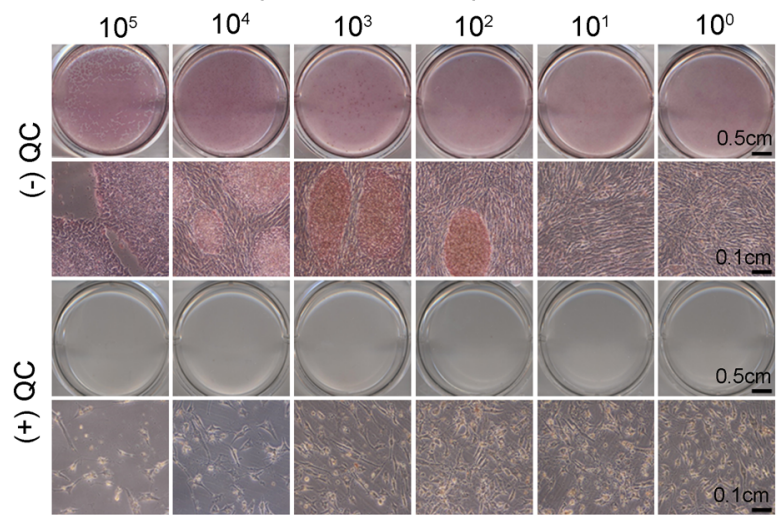

$\mathbf{F}$

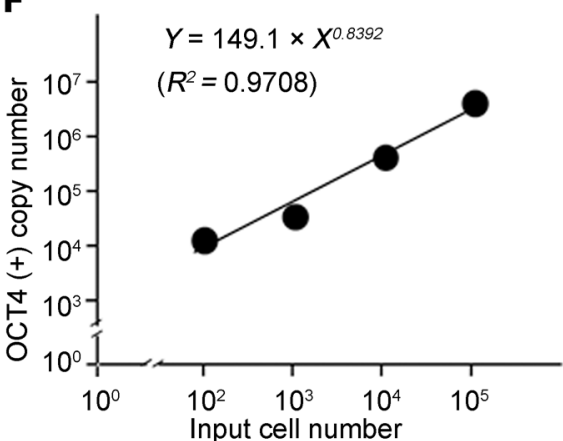

B

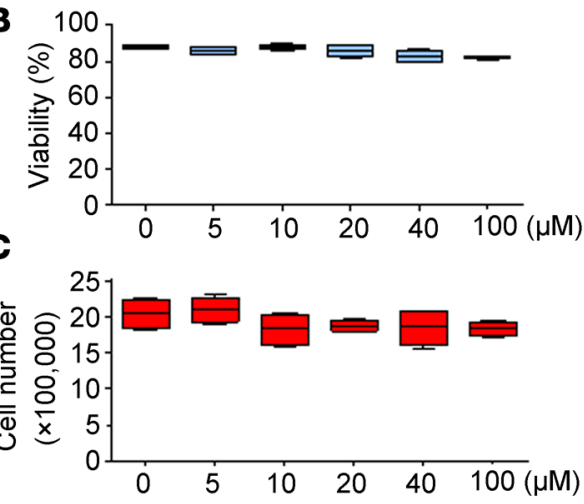

E

\begin{tabular}{|l|c|c|c|c|c|c|c|}
\hline Input & 100,000 & 10,000 & 1,000 & 100 & 10 & 1 \\
\hline Counted & n.c. & 928 & 110 & 7 & 1 & 0 \\
\hline
\end{tabular}

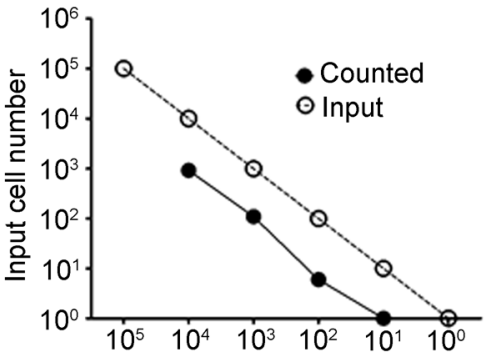

iPS cell numbers (per 100,000 total cells)

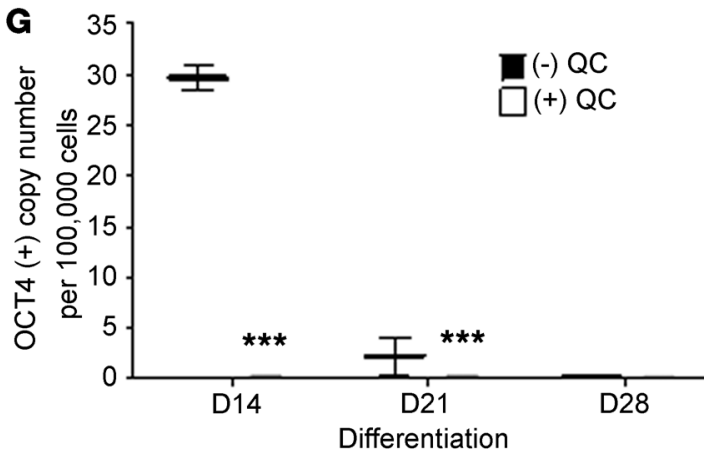

Figure 5. Effects of quercetin treatment on undifferentiated and differentiated cells. (A) Screening to determine optimal quercetin treatment conditions. Surviving hiPSCs were counted using a hemocytometer after treatment with different quercetin concentrations and durations. (B and C) Viability (B) and total cell number (C) of dopaminergic cells at d11 after quercetin treatment on d9. Cultures were treated for 16 hours at 5, 10, 20, 40, and 100 $\mu \mathrm{M}$. Mean $\pm \mathrm{SD} . n=4$. One-way ANOVA. (D) Colony formation by hiPSCs serially diluted by factors of 10 from $10^{5}$ to 1 together with a constant number of fibroblasts $\left(10^{5}\right)$. Cells were treated with $40 \mu \mathrm{M}$ quercetin for 16 hours or left untreated, and then cultured for 6 days, followed by staining for AP activity. Representative results from 2 separate experiments. (E) Plotting of final colony number counted against original input hiPSC number. (F) Generation of standard curve for OCT4 copy number against input hiPSC number by qRT-PCR. OCT4 copy number was measured by qRT-PCR and calculated from 10-fold serially diluted hiPSCs, from $10^{5}$ to $10^{2}$ cells. (G) Measurement using OCT4 qRT-PCR of OCT4-positive cell numbers among mDA cells differentiated from hiPSCs at various time points with or without quercetin treatment. Mean \pm SD. $n=2 .{ }^{* *} P<0.005$, 2-way ANOVA.

The distribution of minor allele fractions of all somatic mutations found across all hiPSC lines (Figure 3C) showed that both clonal and subclonal mutations were observed in each hiPSC line (identified as 2 peaks in plots), but subclonal mutations were unique to individual hiPSC lines. We observed 20 mutations conserved across 2 or more hiPSC lines, but visual inspection of aligned short reads from WES revealed 1 or 2 short reads with mutant alleles in the parent fibroblast for 14 somatic mutation candidates, suggest- ing potential germline origin of these subclonal mutation candidates. Notably, there were no clonal or subclonal somatic mutations in cancer-driver genes such as TP53 in our hiPSC lines (28). C4 and N3 had the lowest somatic mutational burden among the 4 hiPSC lines and were further characterized.

A novel "spotting" culture method reliably generates high-yield and high-quality $m D A$ cells. Numerous laboratories have investigated the in vitro differentiation of mouse and human PSCs 
A

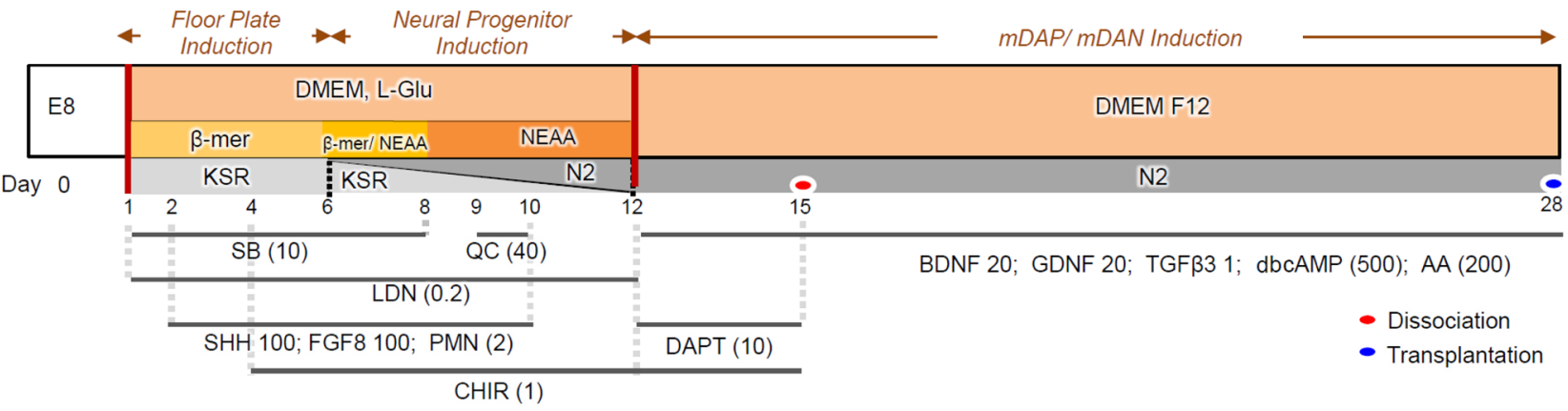

B

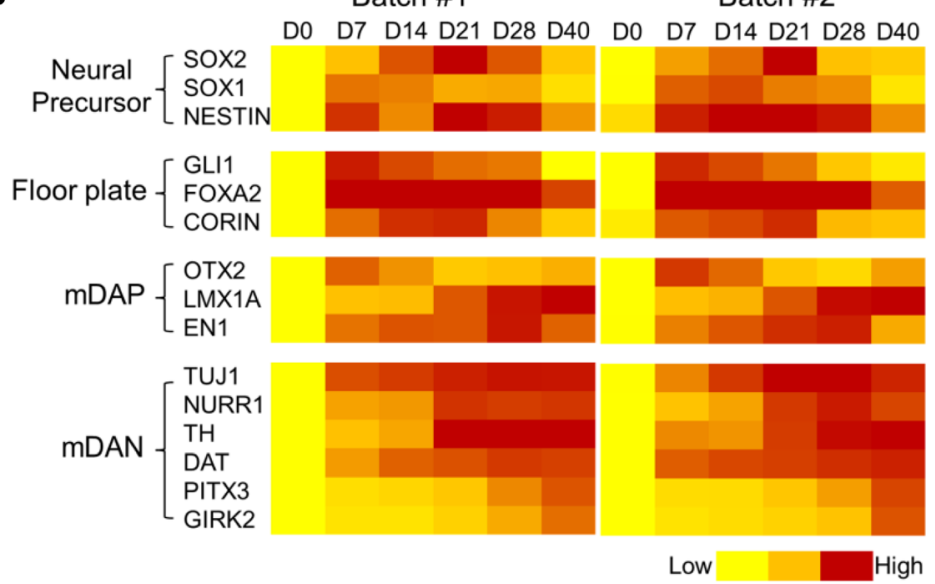

C

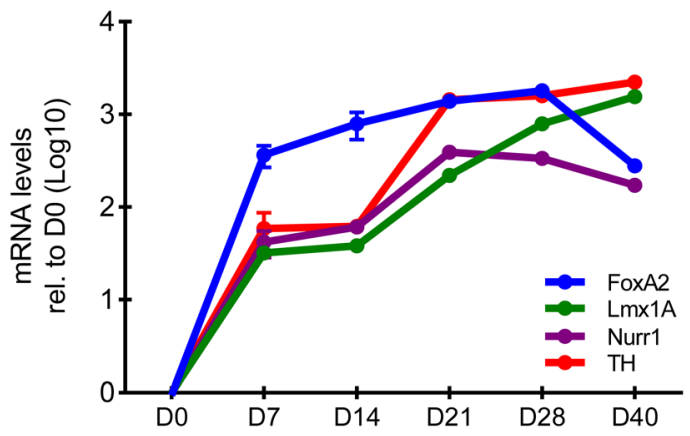

D

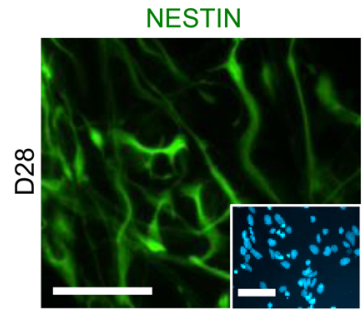

$\mathbf{E}$

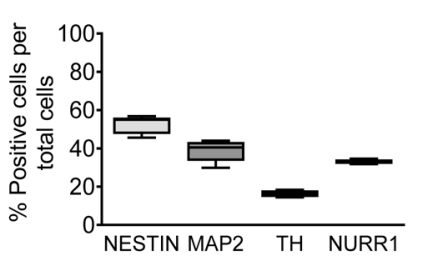

MAP2

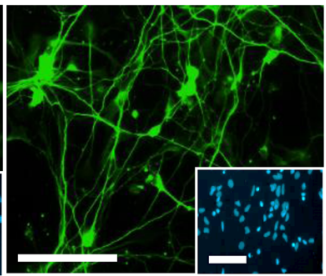

$\mathbf{F}$

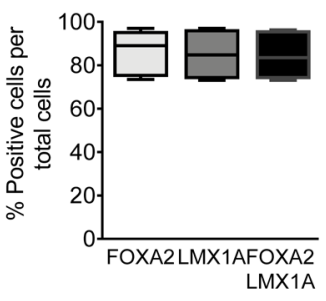

FOXA2/LMX1A/TH

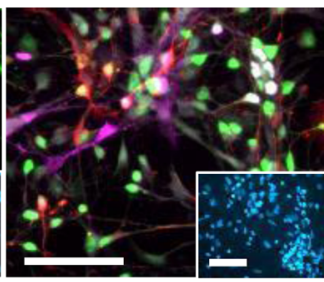

G

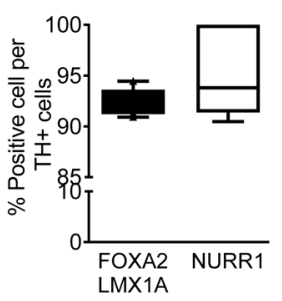

NURR1/TH

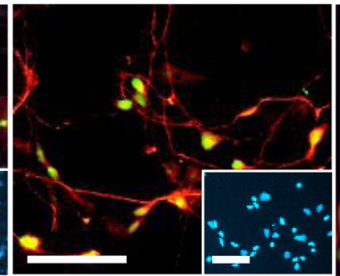

H

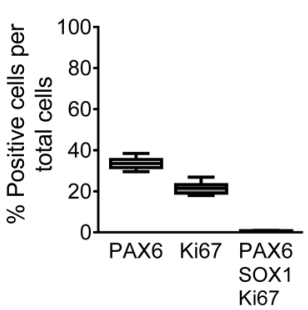

I
PAX6/Ki67/SOX1
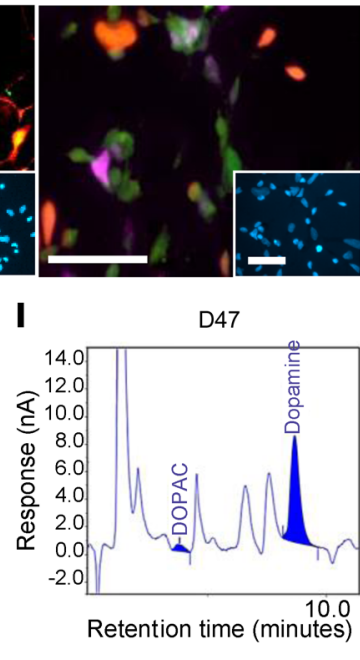

Figure 6. Molecular, cellular, and physiological characterization of in vitro-differentiated C4 hiPSCs. (A) Schematic overview of mDA differentiation method based on spotting protocol. Numbers represent concentrations in $\mathrm{ng} / \mathrm{ml}$, and those in parentheses show $\mu M$. AA, ascorbic acid; $\beta$-mer, $\beta$-mercaptoethanol; BDNF, brain-derived neurotrophic factor; CHIR, CHIR99021; dbcAMP, dibutyryl cyclic adenosine monophosphate; FGF8, fibroblast growth factor 8; GDNF, glial cell line-derived neurotrophic factor; KSR, knockout serum replacement; LDN, LDN193189; L-Clu, L-glutamine; NEAA, nonessential amino acid; PMN, purmorphamine; QC, quercetin; SB, SB431542; SHH, sonic hedgehog. (B) Heatmap of gene expression of stage-specific neural markers in mDA-differentiated cells. (C) Gradual increase in FOXA2, LMX1A, NURR1, and TH gene expression during differentiation. (D) Immunofluorescence staining of neural precursor marker (NESTIN), mDAP markers (FOXA2/LMX1A/TH), mDAN markers (MAP2, NURR1/TH), and proliferating marker (PAX6/SOX1/KI67) cells in differentiated d28 cells. Scale bars: $100 \mu \mathrm{m}$. (E) Percentages of NESTIN+, MAP2 ${ }^{+}, \mathrm{TH}^{+}$, and NURR1 ${ }^{+}$cells among total d28 cells $(n=6)$. (F) Percentag-

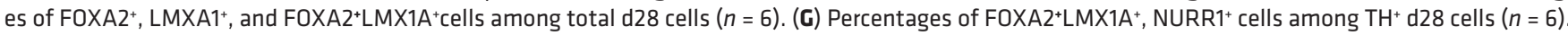

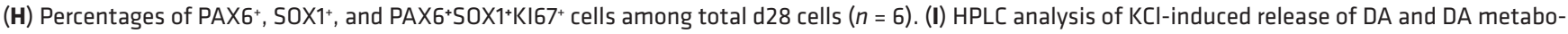
lites (DOPAC) on d47. Data are presented as mean \pm SEM. 
toward the mDA cell fate. Based on the findings that mDANs originate from the neurogenic floor plate and that the Wnt and Shh signals play critical roles (31-33), recent mDA differentiation protocols utilize activators of these signals $(7,34,35)$. Since the embryoid body-derived neurosphere-based method is highly variable between experiments $(18,35,36)$, we sought to establish more efficient and reproducible monolayer methods based on dual-SMAD inhibition $(36,37)$. mDA cells used for transplantation studies have generally been differentiated in vitro for 16 to 32 days (7). Therefore, we sought to optimize the first 15 days, which critically determine floor plate-based mDA progenitor (mDAP) induction, first using the well-studied $\mathrm{H} 9$ (passage number $<36$ )

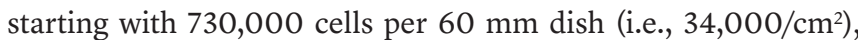
according to previously published optimized conditions (37). Surprisingly, we observed severe cell death/loss starting from $\mathrm{d} 8$ to $\mathrm{d} 10$, resulting in highly variable outcomes. Assessing multiple experiments ( $n=76$ for hESCs and $n=48$ for hiPSCs) performed by 4 independent researchers in our laboratory, more than $50 \%$ failed to provide meaningful data for both hESCs and hiPSCs, due to severe cell loss (Supplemental Figure 5A). Thus, we carefully monitored cell loss during the differentiation process by determining the number of detached cells using FACS during media change (Figure 4A). On d15, we counted the total harvested cell number, then further characterized these cells. Remarkably, the total numbers of detached/lost cells from d1 to d14 were much higher than those of harvested cells (Figure 4B and Supplemental Table 5) when tested for 2 hESC lines (H9 and H7) and 2 hiPSC lines (C4 and N3). Thus, we attempted to start the monolayer culture with smaller numbers of H9 and C4 cells (240,000 [11,000 cells $\left./ \mathrm{cm}^{2}\right]$ and 60,000 [3500 cells $\left.\left./ \mathrm{cm}^{2}\right]\right)$. Using 11,000 cells $/ \mathrm{cm}^{2}$, we found a similar pattern of significant cell loss (Supplemental Table 5). At still lower densities of 3500 cells $/ \mathrm{cm}^{2}$, both $\mathrm{H} 9$ and C4 cells showed poor viability and were similarly lost to detachment, so that the final cell harvest was unacceptably low. This pattern of severe cell loss regardless of initial cell concentration suggests that evenly distributed monolayer conditions are not ideal for in vitro differentiation of hiPSCs and hESCs. Thus, we hypothesized that dividing the monolayer into smaller isolated portions (here called spotting) might improve in vitro differentiation. To test this, we limited initial cell attachment to designated areas by precoating circular areas (spots) of approximately $5 \mathrm{~mm}$ diameter using $10 \mu \mathrm{l}$ of Matrigel on cross points of a $2 \times 2 \mathrm{~cm}$ grid (Supplemental Figure $5, \mathrm{~B}$ and $\mathrm{C})$. To find the optimal cell density, we plated 3 different numbers of cells (40,000,10,000, and 2500) using H9 or C4 cells on each spot. Remarkably, this spotting method substantially reduced cell loss and improved yield at d15 compared with monolayer methods. In particular, we found that 10,000 cells per spot (total of 60,000 cells per $60 \mathrm{~mm}$ dish) resulted in almost $100 \%$ successful in vitro differentiation (Supplemental Figure 5A) and in a final harvest of 6 to 8 million mDA cells at d15, while total cell loss was under 3 million cells (Figure 4B and Supplemental Table 5). We confirmed a similar pattern for H7 hESC and N3 hiPSC lines (Figure 4B), suggesting that this spotting method is broadly applicable to mDA differentiation of hPSC lines. More importantly, cells harvested at d15 included fewer dead cells (Figure 4C) and significantly fewer cleaved caspase-3-positive cells as well as decreased nuclear condensation (Figure 4D), well-known markers for programmed cell death $(38,39)$. We speculated that the difference in outcomes between the spotting and monolayer methods was due to insufficient oxygen and nutrition for cells in monolayer conditions and thus attempted to correct this with more frequent media changes. However, daily media changes neither reduced cell loss nor enhanced cell harvest. On the contrary, this actually increased cell loss (Supplemental Figure 6A). In the spotting method, daily media changes did not affect cell loss or harvest, again confirming that differentiation is more stable with spotting than with the monolayer method. Notably, we observed that the culture media became significantly acidic only in the monolayer culture regardless of frequency of media change (Supplemental Figure 6B), at least in part explaining the poor cell health. Taking these data together, this spotting-based method reduced cell loss, increased final cell yield, and produced healthier mDA cells compared with conventional monolayer methods.

Quercetin treatment eliminates undifferentiated cells during in vitro differentiation. The most critical issue for hPSC-based cell therapy is to establish safety by removing residual undifferentiated cells with neoplastic potential. Based on the previous finding that BIRC5 (encoding survivin) is highly expressed in hPSCs compared with somatic cells (40), we hypothesized that chemical inhibition of survivin would selectively eliminate remaining undifferentiated hiPSCs. However, since survivin is known to be important for neuronal precursors $(41,42)$, it is important to test whether this strategy interferes with mDAP generation. Among survivin inhibitors (40), we chose the flavonoid quercetin $\left(3,3^{\prime}, 4^{\prime}, 5,7\right.$-pentahydroxyflavone) because this natural compound is present at high concentrations in commonly consumed vegetables and fruits (43). We first treated 100,000 undifferentiated C4 cells with 5, 10, 20, 40 , and $100 \mu \mathrm{M}$ quercetin for $2,6,16$, and 24 hours. After washing with fresh media, cells were further cultured for a total of 48 hours. As shown in Figure 5A, viable cells were undetectable when treated with more than $20 \mu \mathrm{M}$ quercetin for more than 16 hours, indicating that undifferentiated hiPSCs can be eliminated with an efficiency of greater than $99.99 \%$. To test whether quercetin affects survival of mDAPs, we treated d9 C4 cells (mostly neuronal precursors) with different concentrations of quercetin for 16 hours and examined outcome on $\mathrm{d} 11$. Neither cell viability nor number was affected at d11 (Figure 5, B and C), suggesting that quercetin does not affect hiPSC-derived mDAPs.

To establish a sensitive and specific assay, we created test mixtures of undifferentiated $\mathrm{C} 4$ cells among hDFs in a total of 100,000 cells and performed 3 different assays. First, we used FACS with monoclonal antibodies against SSEA-4 and TRA-160 (Supplemental Figure 7A) and found a significant discrepancy between the input and the detected cell number, in particular below 100 cells (Supplemental Figure 7B), indicating that this method was insensitive to small numbers of undifferentiated cells. Second, we cultured samples of diluted cells for 6 days and counted AP-positive colonies as a surrogate marker for undifferentiated cells (Figure 5D). Although there was a linear relationship, the number of AP-positive colonies was about one-tenth that expected, given the input cell numbers (Figure 5E). This discrepancy may be due to limited survival and growth of individual hiPSCs and/or to their tendency to aggregate during colony formation. Despite this limitation, since there were no AP-positive colonies detected 
A

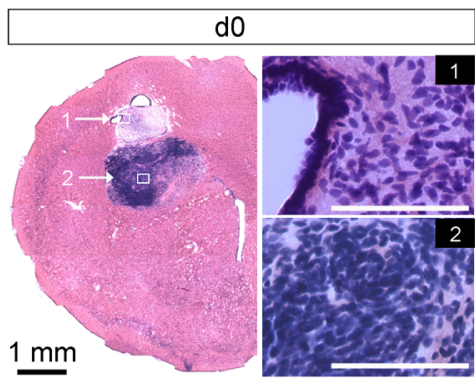

B

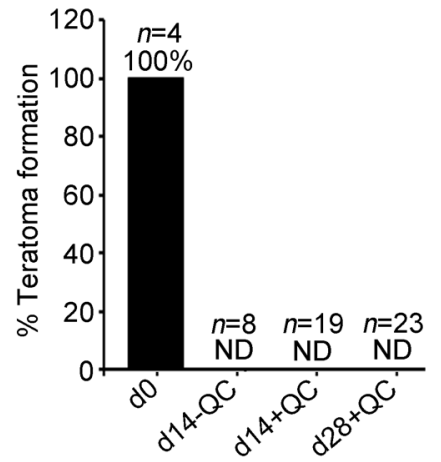

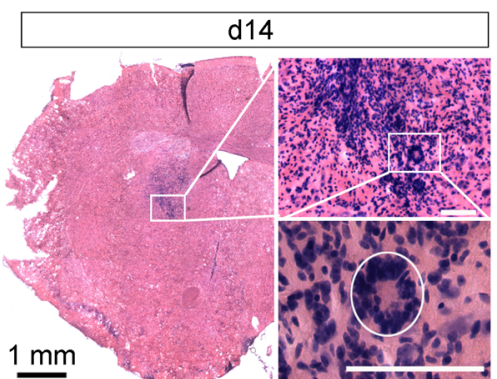
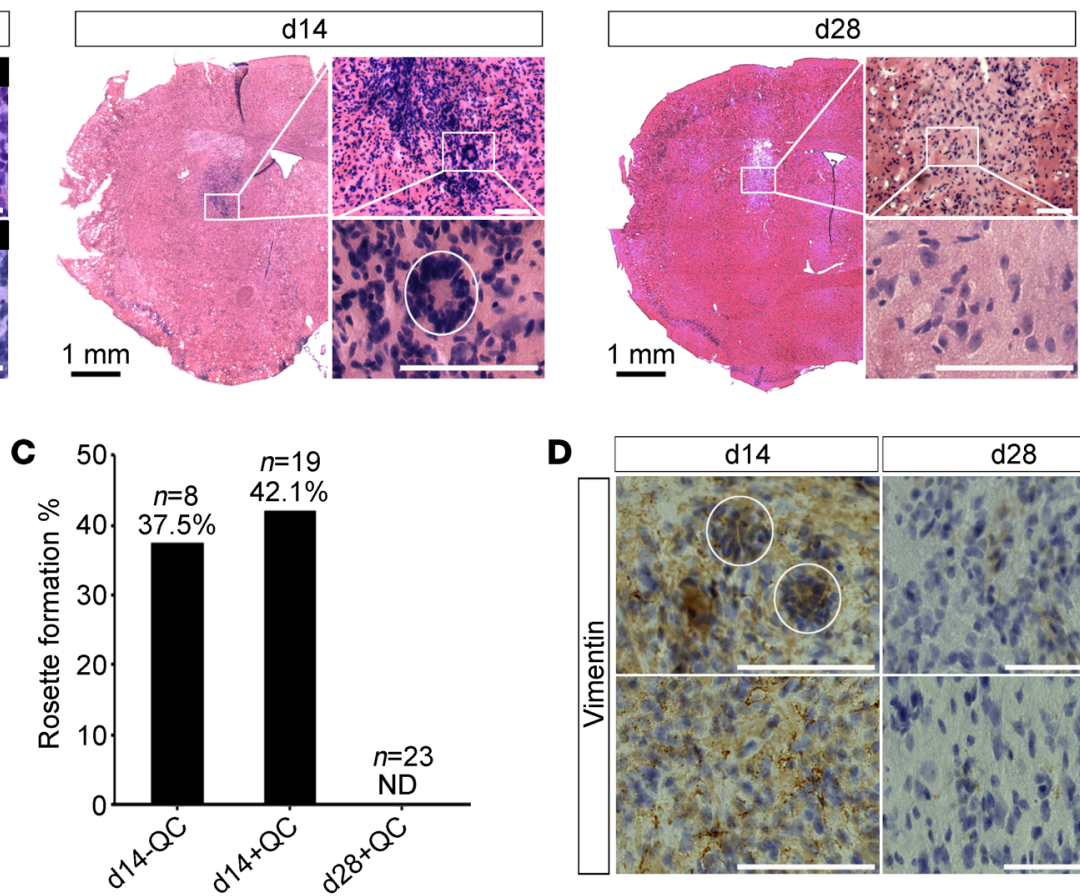

D

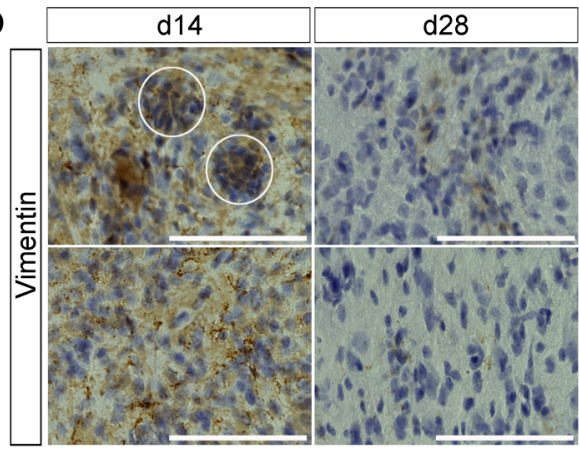

E

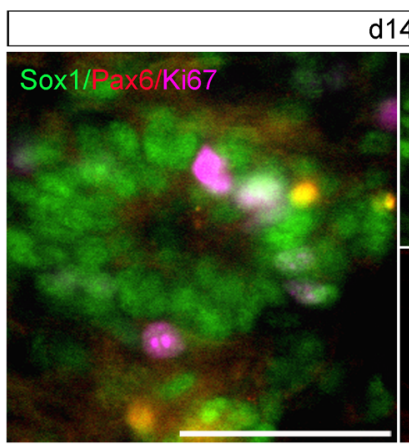

\section{d14}

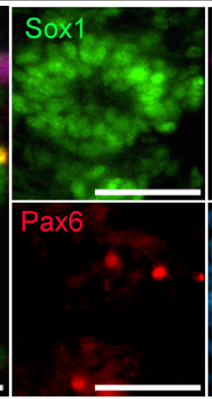

G

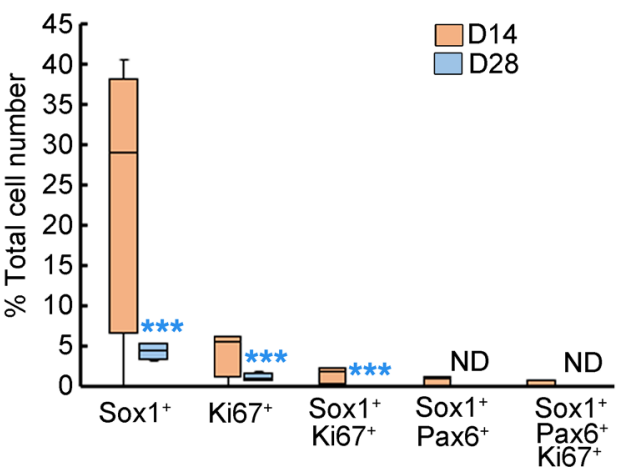

F

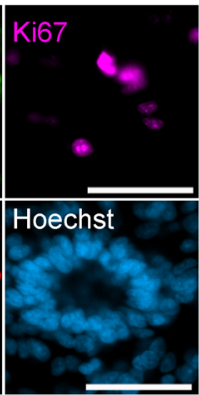

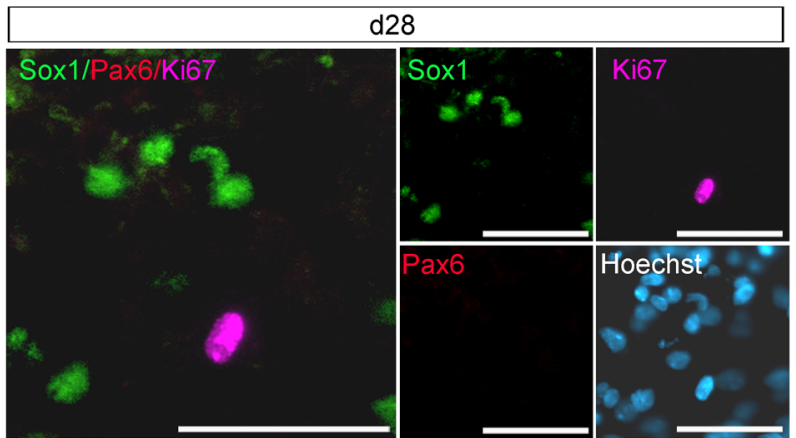

H

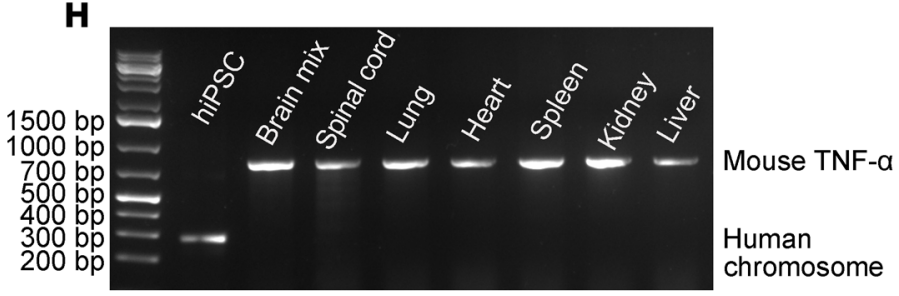

Figure 7. In vivo safety of C4-derived mDA cells in NOD SCID mice. (A) H\&E staining of NOD SCID mouse brain after striatal transplantation of C4 iPS cells (d0, left), or of C4-derived mDAPs at d14 (middle) or d28 (right). The white circle in the d14 group identifies rosette-like structures. (B) Quantification of teratoma formation percentage at d0 $(n=4)$ and d14 without quercetin $(n=4)$ and at d14 $(n=19)$ and d28 $(n=23)$ with quercetin treatment groups. (C) Quantification of rosette formation at d14 of differentiation without quercetin and at d14 and d28 with quercetin treatment. (D) Immunohistochemistry of vimentin in d14 and d28 groups. (E and F) Immunofluorescence staining of SOX1, PAX6, and KI67 in d14 (E) and d28 groups (F). (G) Quantification of SOX1+,

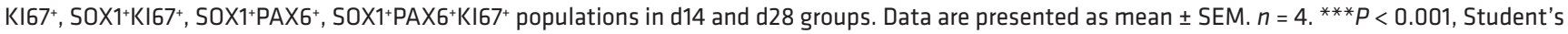
$t$ test. (H) Biodistribution assay. RT-PCR of human- or mouse-specific gene expression in "brain mix" (mixture of olfactory bulb and cerebellum), spinal cord, lung, heart, spleen, kidney, and liver of the NOD SCID mice that had received intrastriatal hiPSC-derived d28 dopaminergic progenitor grafts 6 months previously. hiPSC serves as a positive control. The human-specific gene is located on chromosome 10 at 29125650 to 29125967 . The mouse-specific gene is part of mouse TNF- $\alpha$. Scale bars: $100 \mu \mathrm{m}$ (unless otherwise specified). 
after treatment with quercetin even when $10^{5}$ hiPSCs were plated, this result confirmed that the efficacy of quercetin treatment is greater than $99.99 \%$. Nonetheless, since this method cannot detect numbers fewer than 10 undifferentiated cells per 100,000, we next used a qRT-PCR method using OCT4 expression as a surrogate marker. A standard curve of OCT 4 copy number was generated using qRT-PCR analysis of mRNAs prepared from $10^{2}$ to $10^{5}$ undifferentiated $\mathrm{C} 4$ cells (Figure $5 \mathrm{~F}$ ), allowing us to predict the number of undifferentiated cells. Using this assay, the calculated number of undifferentiated C4 cells at d14, d21, and d28 without quercetin treatment was 30,2 , and 0.17 per 100,000 cells, respectively (Figure 5G). Thus, if 10 million cells at d14, d21, or d28 of differentiation were transplanted into a PD patient, grafts would contain approximately 3000, 200, and 17 undifferentiated cells, respectively. Since quercetin treatment can eliminate undifferentiated cells with greater than $99.99 \%$ efficiency following quercetin treatment, the expected number of undifferentiated cells would be at most $17 \times 0.01 \%=0.0017$ cells per 10 million $\mathrm{d} 28$ cells. In agreement with this, the calculated number of undifferentiated cells using the qRT-PCR curve at d14, d21, and d28 after quercetin treatment ( $40 \mu \mathrm{M}$ at $\mathrm{d} 9$ for 16 hours) was far less than 1 cell per 100,000 cells (Figure 5G). Consistent with these results, a few $\mathrm{NANOG}^{+}$cells were observed at d14 without quercetin treatment, but none were detected with treatment (Supplemental Figure 7C). Taken together, our results indicate that quercetin treatment reduces the number of undifferentiated cells to levels undetectable using sensitive techniques, thus greatly reducing the risk of tumor formation even when many millions of differentiated cells are transplanted.

Functional characterization of $M D A P$ and $M D A N$. Based on the spotting method and quercetin treatment described above, we established a modified in vitro protocol for differentiation of hiPSCs into $\mathrm{mDAP} / \mathrm{mDAN}$ (Figure $6 \mathrm{~A}$ ). C4 cells differentiated using these methods showed progressively more compact morphology, minimal detachment (Supplemental Figure 6C), and bipolar outgrowth of neurites from the edge (Supplemental Figure 8A). At $\mathrm{d} 15$, cells were dissociated into single-cell suspensions, replated, and further differentiated. As shown in Figure 6, B and C, expression of neural precursor markers (e.g., SOX2, SOX1, and NESTIN) and floor/basal plate markers (e.g., GLI1, FOXA2, and CORIN) started at $\mathrm{d} 7$ and continued at high levels through $\mathrm{d} 28$, finally decreasing at $\mathrm{d} 40$. The expression of mDAP markers (e.g., OTX2, LMX1A, and EN1) was elevated at d21 and 28, whereas mDAN markers (e.g., TH, DA transporter [DAT], and PITX3) increased later. These data were corroborated by stage-specific immunocytochemistry (ICC) analyses showing a gradual decrease of NESTIN and increases of mDAN markers (Supplemental Figure $8 B$ ). Since $\mathrm{d} 28$ cells exhibited more mature phenotypes than $\mathrm{d} 14$ cells (Supplemental Figure 8B), we expected that d28 cells might be more suitable than earlier cells and thus analyzed $\mathrm{d} 28$ cells using ICC for typical mDAP and mDAN markers (Figure 6, D-H). Approximately $40 \%$ and $15 \%$ of the total cells expressed MAP2 and $\mathrm{TH}$, respectively, and $38 \%$ of the cells expressed NURR1 (Figure 6, D and E). More than $80 \%$ of the total cells coexpressed FOXA2 and LMX1A (Figure 6, D and F), and the majority of $\mathrm{TH}^{+}$ cells coexpressed FOXA2, LMX1A, and NURR1 (Figure 6, D and $\mathrm{G})$, a feature characteristic of mDA phenotypes. We observed that approximately $30 \%$ and $20 \%$ of $\mathrm{d} 28$ cells expressed the dorsal patterning marker PAX6 and the proliferation marker Ki67, respectively (Figure 6, D and $\mathrm{H}$ ). Importantly, cells that coexpress PAX6, SOX1, and Ki67, known to form abnormal outgrowth upon transplantation $(44,45)$, were undetectable (Figure 6, D and $\mathrm{H}$ ). $\mathrm{GABA}^{+}$or $5-\mathrm{HT}^{+}$cells were barely detected at any stage of differentiation (Supplemental Figure 8B).

To determine whether these cells become physiologically functional neurons, we performed whole-cell patch-clamp recordings from $\mathrm{d} 70$ cultures that contained many $\mathrm{TH}^{+}$neurons coexpressing MAP2, DAT, and synaptophysin (SYP) (Supplemental Figure 9A). These d70 $\mathrm{TH}^{+}$neurons coexpressed additional mature mDA markers, including PITX3 and VMAT2 (Supplemental Figure 9A). Also, $\mathrm{TH}^{+} \mathrm{ALDH} 1 \mathrm{~A} 1^{+}$neurons coexpressed GIRK2 or sometimes CALBINDIN (Supplemental Figure 9B), characterizing A9- and A10-type mDANs, respectively. In current-clamp recoding mode, we assessed the intrinsic membrane properties of these cells (resting membrane potential, $-55.93 \pm 2.43 \mathrm{mV}$; input resistance, $1.52 \pm 0.44 \mathrm{G} \Omega ; n=7$ neurons) and observed action potentials in response to depolarizing current injections (Supplemental Figure 10A; average amplitude of action potential 54.96 $\pm 4.66 \mathrm{mV}$; half-width: $6.04 \pm 0.82 \mathrm{~ms}$; amplitude of afterhyperpolarization [AHP]: $3.55 \pm 1.46 \mathrm{mV} ; n=7$ cells). In voltage-clamp recording mode, voltage pulses from $-70 \mathrm{mV}$ to $+40 \mathrm{mV}$ evoked transient inward currents, which were completely blocked by tetrodotoxin (TTX) (a voltage-gated $\mathrm{Na}^{+}$channel blocker), indicating expression of voltage-gated $\mathrm{Na}^{+}$channels, as well as sustained outward currents (Supplemental Figure 10B), representing potassium currents. Moreover, during whole-cell voltage-clamp recordings, we observed spontaneous postsynaptic currents (sPSC), indicating the presence of functional synapses (at a holding potential of $-70 \mathrm{mV}$, the frequency of sPSCs was $0.11 \pm 0.03 \mathrm{~Hz}$; peak amplitude, $14.71 \pm 3.05 \mathrm{pA}$; rise time, $0.73 \pm 0.14 \mathrm{~ms}$; decay time, $1.72 \pm$ $0.19 \mathrm{~ms} ; n=5$ cells) (Supplemental Figure 10C). Consistent with pacemaker activity at the resting membrane potential (46), spontaneous firing was observed in the absence of an injected current. The recorded cells fired spontaneously, with an average frequency of $4.4 \pm 0.8 \mathrm{~Hz}(n=4)$, as typically observed in A9 mDANs (Supplemental Figure 10D). Individual recorded neurons (loaded with neurobiotin through the recording patch pipette) colocalized with TH positivity, confirming the identity of these cells as dopaminergic neurons (Supplemental Figure 10E). In addition to single-cell patch-clamp recordings, we measured population-level electrical activity using multielectrode array (MEAs). Differentiated cells developed robust synchronous bursting patterns indicative of maturing neuronal networks (Supplemental Figure 10F). Cumulative activity maps showed an increase in the spike density and the area of spikes within mDA between $\mathrm{d} 30$ and $\mathrm{d} 44$ (Supplemental Figure 10F). In order to isolate spontaneous activity from mDANs, the cultures were treated with a combination of glutamate receptor antagonists, $\mathrm{NBQX}+\mathrm{AP} 5$, and $\mathrm{GABA}_{\mathrm{A}}$ receptor antagonist, picrotoxin. When this cocktail was administered, overall spiking and the number of active electrodes were only modestly diminished (Supplemental Figure 10, G and H), suggesting that there are abundant mDANs in these cultures. Finally, HPLC analysis of culture medium further showed that $\mathrm{d} 47$ cells release DA $(3.1 \pm 0.1$ $\mathrm{ng} / \mathrm{ml})$ and DOPAC $(0.2 \pm 0.0 \mathrm{ng} / \mathrm{ml})$ (Figure 6I). 

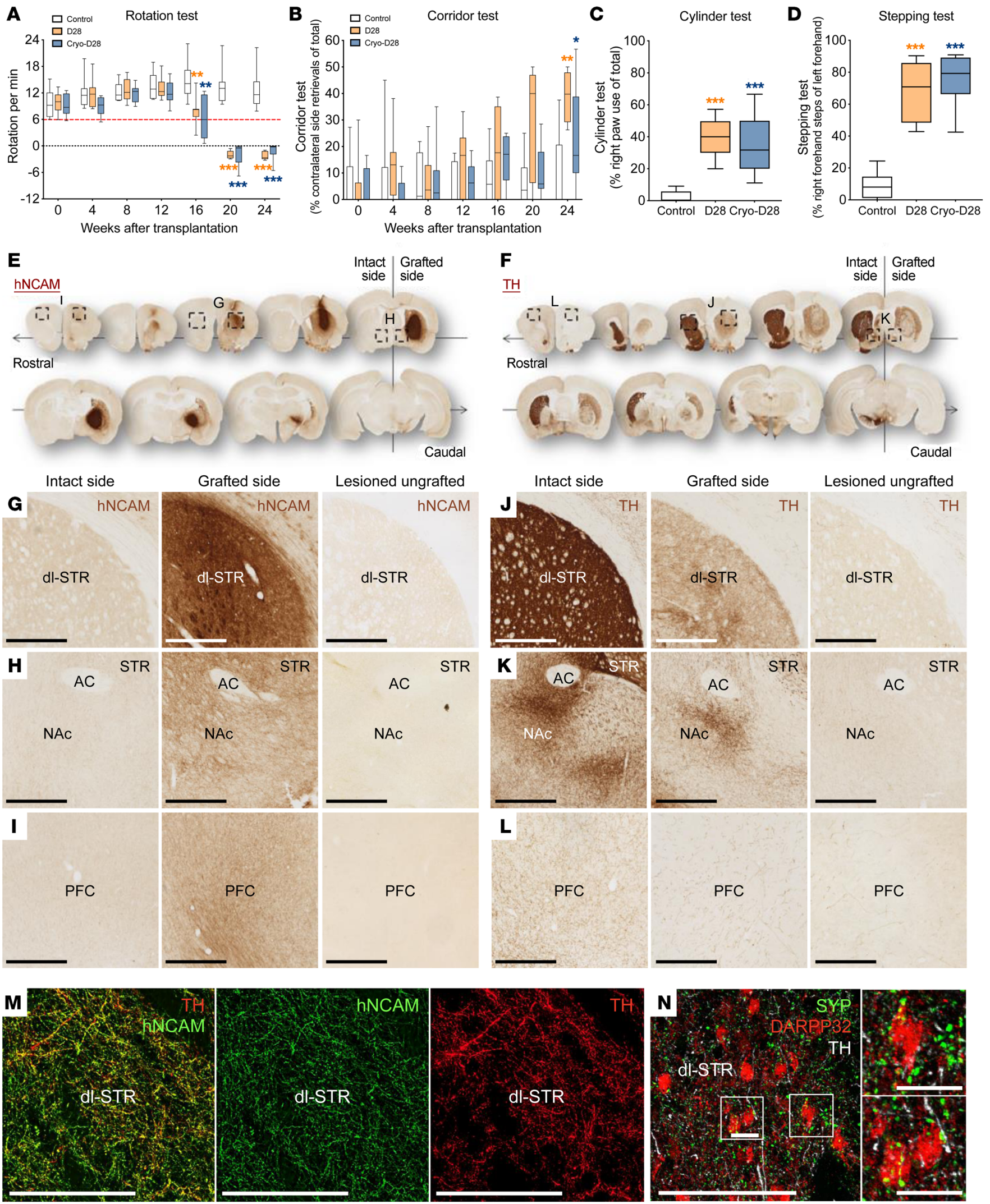

Figure 8. In vivo survival and function of C4 hiPSC-derived mDA cells. (A-D) Behavioral assessments using drug-induced rotation test (A), corridor test (B), cylinder test (C), and stepping test (D) in d28 and Cryo-d28 groups $(n=9)$. ( $(\mathbf{C}$ and $\mathbf{F})$ Overview of graft-derived hNCAM+ innervation and $\mathrm{TH}^{+}$innervation of the host brain. (G-L) Innervation by graft-derived hNCAM+ neurons (G-I) or TH+ neurons $(\mathbf{J}-\mathbf{L})$ into host STR, NAc, and PFC of intact side, grafted side, and lesioned ungrafted side. (M) High magnification image showing graft-derived innervation. (N) Immunofluorescence staining of the human-specific synaptic marker SYP, TH, and DARPP32 in grafted neurons. All graft analysis data (E-M) were obtained 26 weeks after transplantation. AC, anterior commissure; dl-STR, dorsal STR. Data are presented as mean \pm SEM. ${ }^{*} P<0.05 ;{ }^{* *} P<0.01 ;{ }^{* *} P<0.001$, Student's $t$ test. Scale bars: $500 \mu \mathrm{m}(\mathbf{G}-\mathbf{L}) ; 100 \mu \mathrm{m}(\mathbf{M}$ and $\mathbf{N})$; and $20 \mu \mathrm{m}$ for insets in $\mathbf{N}$. 
In vivo safety following transplantation. Our in vitro characterization showed that the majority of cells from $\mathrm{d} 14$ to $\mathrm{d} 28$ represent mDAPs, suitable as transplantable cell sources. To test their safety, we transplanted $\mathrm{d} 14$ or $\mathrm{d} 28 \mathrm{C} 4$ cells (without or with quercetin treatment; 100,000 cells per animal) into the striatum (STR) of immunodeficient NOD SCID mice. As expected, transplantation of undifferentiated $\mathrm{C} 4$ cells (d0) induced formation of teratomas containing the characteristic 3 germ layers in all 4 mice tested (Figure 7A), a defining characteristic of PSCs. By comparison, no teratoma formation was observed when $\mathrm{d} 14$ cells without $(n=8)$ or with quercetin ( $n=19$, Figure 7A) and d 28 cells with quercetin ( $n$ $=23$; Figure 7A) treatment were transplanted (Figure 7B). Interestingly, we observed rosette-like structures in about $40 \%$ of the host brains grafted with $\mathrm{d} 14$ cells ( 3 of 8 in d 14 without quercetin group; 8 of 19 in d14 with quercetin group; Figure 7A). In contrast, there were no rosette-like structures when $\mathrm{d} 28$ cells were transplanted (0 of 23 mice; Figure 7, A and C). Immunohistochemistry showed that, compared with $\mathrm{d} 28$ grafts, d14 grafts contained more vimentin-positive immature cells (Figure 7D). In addition, SOX1 positive, KI-67 positive, SOX1/KI-67 double-positive, SOX1/PAX6 double-positive, and SOX1/PAX6/KI-67 triple-positive cells were also fewer in grafts from $\mathrm{d} 28$ cells than in those from d14 cells ( $33 \%$ vs. $4.5 \% ; 5.9 \%$ vs. $1.2 \% ; 2.1 \%$ vs. $0.15 \% ; 1.2 \%$ vs. not detected [ND]; $0.75 \%$ vs. ND, Figure 7, E-G), indicating that d28 grafts contain fewer cells with proliferative potential than $\mathrm{d} 14$ grafts. These results suggest that, although fully undifferentiated cells had been removed and teratomas were not formed, d14 grafts still contained immature progenitor cells capable of forming rosettelike structures. Additionally, since SOX1/PAX6/KI-67 triple-positive cells were undetectable in $\mathrm{d} 28$ grafts, we conclude that $\mathrm{d} 28$ cells represent a safer cell source for transplantation than $\mathrm{d} 14$ cells. We further tested the safety of $\mathrm{d} 28$ cells by assessing their biodistribution. At 6 months following transplantation of $\mathrm{d} 28$ cells into the STR, we harvested central nervous system regions (mixtures of olfactory bulb and cerebellum and spinal cord) and 5 peripheral organs (lung, heart, liver, kidney, and spleen) to search for migration of human-origin cells from the striatal grafts. Genomic qPCR detected no human DNA sequences in any of these regions, while hiPSC-positive controls showed prominent expression (Figure $7 \mathrm{H}$ ), demonstrating no detectable redistribution of grafted cells within the brain or to peripheral organs.

In vivo efficacy tests and graft analyses in animal models of $P D$. The 6-OHDA lesioned rat model was historically the first PD animal model developed $(47)$ and remains a popular model $(48,49)$. It is especially useful for quantitative assessment of motor effects of cell transplantation. Its use in athymic rats is emerging as the preferred model because immunosuppression is unnecessary. Two different sources of athymic rats (Taconic Biosciences and Charles River) were used. We first transplanted 100,000 and 300,000 C4 d28 cells to the STR of unilaterally 6-OHDA-lesioned athymic Taconic rats and monitored their amphetamine-induced rotation behavior monthly after transplantation. At 12 weeks, both the 100,000- and the 300,000-cell groups showed significant reduction of ipsilateral rotation behavior (Supplemental Figure 11A). At 16 weeks, rotation behavior was completely rescued in all implanted rats, and some even showed contralateral rotation. In contrast, rats receiving vehicle showed no recovery. H\&E staining showed that grafts contained neither teratoma nor rosettes (Supplemental Figure 11B). Immunohistochemistry for human neural cell adhesion molecule (hNCAM) showed dense $\mathrm{hNCAM}^{+}$innervation in the STR (Supplemental Figure 11C), prefrontal cortex (PFC) (Supplemental Figure 11D), septal nuclei (Supplemental Figure 11E), nucleus accumbens (NAc) (Supplemental Figure 11F), and corpus callosum (CC) (Supplemental Figure 11G). Immunohistochemistry revealed abundant $\mathrm{TH}^{+}$neurons in the graft (Supplemental Figure $11 \mathrm{H}$ ). Stereological quantification showed that the average number of surviving $\mathrm{TH}^{+}$neurons was 5,621 \pm 1029 per 100,000 grafted cells $(n=4)$, containing a mixture of neuronal morphologies including large, angular cell somata typical of A9 neurons (Supplemental Figure 11I) and smaller spherical neurons typical of A10 identity (Supplemental Figure 11J).

With these data suggesting safety and efficacy of d28 C4 cells, we further extensively tested them in athymic rats from Charles River, which were more physically robust and facilitated longer-term analyses than the Taconic strain. We selected a single dose (100,000 cells) of d28 C4 cells for transplantation (Supplemental Figure 11K) and compared the efficacy and safety of cryopreserved to freshly prepared cells. Cryopreserved d28 C4 cells (Cryo-d28) retained a level of viability similar to that of freshly prepared equivalents (approximately 90\%) and displayed the same mDA cell phenotypes (Supplemental Figure 11L) following storage for 1 week in liquid nitrogen. Amphetamine-induced rotation was significantly reduced at 16 weeks and completely rescued at 20 and 24 weeks after transplantation of either fresh or Cryo-d28 C4 cells (Figure 8A). As noted for the Taconic rats (Supplemental Figure 11A), some animals exhibited contralateral rotation at 20 and 24 weeks. We also evaluated the functional efficacy of these grafts in several tests not involving exogenous pharmacological stimulation, thus providing a measure of motor deficits more analogous to those of human PD. In the corridor test, a sensitive test of lateralized sensorimotor response selection (50), either fresh or Cryo-d28 C4 cells significantly reduced the lesion-induced ipsilateral bias at 24 weeks after transplantation (Figure 8B). Notably, no significant reduction had been observed yet at 16 or 20 weeks, suggesting that improvement in this task takes more time than rotation behavior. In the cylinder and stepping tests, which measure forelimb akinesia (51, 52), impaired forelimb function produced by 6-OHDA lesion was also significantly improved by transplantation of fresh or Cryo-d28 C4 cells at 24 weeks after transplantation (Figure 8, C and D). Taking these data together, in all 4 behavioral tests, both fresh and Cryo-d28 C4 cells significantly and similarly improved motor dysfunction. Further, additional grafted animals at later time points demonstrated that recovery of rotation behavior was sustained up to 52 weeks (Supplemental Figure 11M), the latest time tested, suggesting that functional improvements due to transplantation are well maintained.

Since H9-derived mDA cells have been extensively validated and explicitly shown to be equally functional to human fetal ventral mesencephalic (VM) cells (53), we directly compared outcomes following transplantation of H9 hESC- and C4 hiPSC-derived d 28 cells. Transplantation of $1 \times 10^{5}$ and $4 \times 10^{5}$ cells showed that both lines resulted in identical recovery of rotational behavior both in degree and in time course (Supplemental Figure 12A). 
We next analyzed grafts at 26 weeks after transplantation and found that both $\mathrm{hNCAM}^{+}$and $\mathrm{TH}^{+}$cells displayed extensive innervation of the entire STR with copious extension to dopaminergic target areas such as dorso lateral STR (dl-STR), PFC, and NAc (Figure 8, E-L, and Supplemental Figure 12B). Robust innervation of the host brain by these graft-derived mDANs was further validated by extensive coexpression of hNCAM in dopaminergic fibers in the dl-STR (Figure 8M). In addition, triple-immunofluorescence staining was performed using antibodies against $\mathrm{TH}$, a human presynaptic protein (SYP), and a striatal medium spiny neuron marker (DARPP32). We observed $\mathrm{TH}^{+} \mathrm{SYP}^{+}$neuronal terminals on their preferential targets and the host dendritic spines (DARPP32 $2^{+}$neurons) at the border of the grafts, indicating that grafted DANs had formed synaptic connections with the host striatal neurons (Figure $8 \mathrm{~N}$ ). Cryo-d28 and fresh $\mathrm{d} 28$ grafts showed similar $\mathrm{hNCAM}^{+} \mathrm{TH}^{+}$reinnervation and synaptic formation patterns (Supplemental Figure 12C). Grafts of both fresh and Cryo-d28 C4 cells contained similarly high numbers of DA neurons with A9- or A10-like morphologies

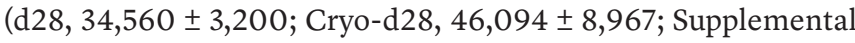
Figure 13, A and B). The graft volume was also similar between $\mathrm{d} 28$ and Cryo-d28 (d28, $12.2 \pm 1.1 \mathrm{~mm}^{3}$; Cryo-d28, $13.0 \pm 1.7$ $\mathrm{mm}^{3}$; Supplemental Figure 13C). The total number of $\mathrm{hNCAM}^{+}$ cells was approximately $3.0 \times 10^{6}$ and $2.45 \times 10^{6}$, and the average percentage of $\mathrm{TH}^{+}$cells was $1.48 \% \pm 0.55 \%$ and $2.08 \% \pm 0.65$ $\%$ in the grafts of $\mathrm{d} 28$ and Cryo-d28, respectively. Most (70\%$80 \%)$ of these $\mathrm{TH}^{+}$neurons coexpressed FOXA2 and LMX1A, and more than $90 \%$ coexpressed NURR1 (Supplemental Figure 13, D-F). A mature DA marker, DAT, was abundantly expressed in $\mathrm{TH}^{+}$neurons (Supplemental Figure 13G), while a marker associated with proliferative potential, $\mathrm{KI} 67^{+}$, was expressed in less than $1 \%$ of cells (d28, $0.86 \pm 0.09 \%$; Cryo-d28, $0.54 \pm 0.21 \%$; Supplemental Figure 13, H and I). Rosettes or teratomas were not observed, and proliferative cells coexpressing SOX1, PAX6, and Ki67 were scant or undetected (SOX1 ${ }^{+} \mathrm{PAX}^{+}$, d28, $0.37 \%$ \pm 0.10\%; Cryo-d28, 0.15\% \pm 0.11\%; SOX1 ${ }^{+}$PAX6 ${ }^{+} \mathrm{KI}^{6} 7^{+}, \mathrm{d} 28$, $0.02 \% \pm 0.02 \%$; Cryo-d28, N/D; Supplemental Figure $13, \mathrm{H}$ and I). GIRK2 or CALBINDIN was expressed in $\mathrm{TH}^{+}$neurons (Supplemental Figure 13, J and $\mathrm{K}$ ), with the majority coexpressing GIRK2 (d28, 79.29\% $\pm 4.88 \%$; Cryo-d28, 81.28\% $\pm 3.50 \%$; Supplemental Figure 13L). These $\mathrm{TH}^{+}$neurons in grafts coexpressed additional A9 markers, such as ALDH1A1. $\mathrm{TH}^{+} \mathrm{ALDH} 1 \mathrm{~A} 1^{+}$ neurons often coexpressed SOX6 and GIRK2, representing A9-type mDANs (Supplemental Figure 13, M and N); while some $\mathrm{TH}^{+} \mathrm{ALDH} 1 \mathrm{~A} 1^{+}$neurons coexpressed CALBINDIN, representing A10-type mDANs (Supplemental Figure 13O). In sum, these data show that the great majority of $\mathrm{TH}^{+}$neurons in grafts of both fresh and Cryo- $\mathrm{d} 28 \mathrm{C} 4$ cells have the characteristics of A9-type mDANs, consistent with the extensive and long-term recovery of motor dysfunction in behavioral tests.

Finally, we tested the scalability and clinical applicability of our platform by production and characterization of differentiated C4 cells in vitro under this protocol at the Good Manufacturing Practice (GMP) facility at the Dana Farber Institute. We successfully produced more than 160 million d28 cells starting from about 1 million dO C4 iPS cells (Supplemental Figure 14). Quality control data (e.g., genomic footprint, ICC of marker proteins, qRT-PCR) demonstrated that these clinically relevant quantities were free of pathogens and of high quality, as evidenced by the high percentage of FOXA2 ${ }^{+} \mathrm{LMX}^{+} \mathrm{A}^{+}$cells (>85\%) and absence of inappropriate markers (e.g., 5-HT, DBH, OCT4, and SSEA4; representing serotonergic, noradrenergic, and pluripotent markers, respectively).

\section{Discussion}

PD is a particularly promising target for cell-replacement therapy because selective degeneration of a well-characterized cell type - A9-type mDANs - is the major cause of motor dysfunction associated with the condition. Numerous researchers have extensively investigated cell therapy for PD using diverse cell sources, including fetal tissues, autologous adult stem cells, and allogeneic mDA cells $(5-7,54)$. We have focused instead on hiPSC-derived autologous cell replacement because of its unique advantages in mitigating ethical, practical, and medical issues. To help realize the potential of personalized autologous cell therapy for PD, we sought to address current technical and scientific barriers to the implementation of this therapeutic strategy.

Because personalized cell therapy would require generation of clinical-grade hiPSCs from each patient treated, it is critical to establish reprogramming technology allowing efficient and reliable generation of such lines. We found that a combination of 2 metabolism-modulating miRNAs (miR-302s and miR-200c) with the canonical Yamanaka factors (Y4F) facilitated the generation of hiPSCs meeting stringent quality criteria. First, our hiPSC lines showed expression levels of authentic pluripotency markers, including OCT4, SOX2, NANOG, ESRRB, REX1, GDF3, ECAT1, GBX2, and TRA-1-60, similar to those of H9 (Figure 2, A and B). Second, H9 hESC and hiPSC lines generated by $\mathrm{Y} 4 \mathrm{~F}+3+2$ (our final method), but not by $\mathrm{Y} 4 \mathrm{~F}$ or $\mathrm{Y} 4 \mathrm{~F}+3$, differentiated equally well to all 3 germ layer lineages, as determined by immunostaining and gene expression of 3 germ layer-specific markers (Figure 2, C and D). Third, our hiPSC lines derived from multiple hDFs showed typical hESC-like compact colony morphology with well-defined borders (Supplemental Figures 2A and 3A). The robustness of this method was validated by successful generation of multiple hiPSC lines from 13 different adult hDF sources. How this same combination would perform using alternative delivery methods (e.g., mature RNA/miRNA or Sendai virus) and in other cell types (e.g., blood and urine cells) warrants further investigation.

The most important criteria of hiPSC-based therapy are long-term safety and efficacy following cell transplantation. The genomic integrity of hiPSCs should be established before they are used for therapy. For example, Merkle and colleagues reported that several hESC lines, including H9, develop mutations in the TP53 gene encoding the tumor-suppressor P53, which are mutations commonly seen in human cancers (28). Notably, in the first hiPSC-based human trial, hiPSCs derived from 1 of 2 patients were found to have a minor cancer-causing mutation, resulting in cancellation of this second patient's cell treatment (17). To confirm genomic integrity, we analyzed 5 independent hiPSC lines derived from a sporadic PD patient (MCL540 in Supplemental Table 2) by karyotyping, qRT-PCR, and WES analyses and found that 4 clones $(\mathrm{C} 4, \mathrm{~N} 3, \mathrm{C} 11, \mathrm{C} 5)$ out of 5 were free of integrated plasmid DNAs and contained no somatic mutations causally implicated in cancer, showing that our reprogramming method can reli- 
ably generate clinically viable hiPSC lines. These 4 hiPSC clones contained significantly fewer variants per line, compared with 140 hESC lines studied by Merkle and colleagues (28). In particular, they contained significantly fewer coding variants in genes reported in the COSMIC database (Figure 3). Another critical safety issue facing hiPSC-based therapy is the need to eliminate the neoplastic potential of residual undifferentiated cells. In this study, we established a chemical method using quercetin, targeting hPSC-specific BIRC5 (40), that eliminated undifferentiated PSCs with greater than $99.99 \%$ efficiency (Figure 5). Our theoretical calculation based on qRT-PCR analysis of OCT4 expression predicts 0.0017 undifferentiated cells per 10 million d28 cells after quercetin treatment. Given that the spontaneous incidence rates for all types of glioma range from 4.67 to 5.73 per 100,000 (55), the risk of tumor formation would thus compare favorably to the spontaneous incidence of gliomas. We emphasize that our chemical method is simple, effective, and requires no additional handling, such as cell sorting or $\gamma$-ray irradiation $(45,56)$, and thus can readily meet GMP standards. However, quercetin treatment does not directly eliminate neural overgrowth from rosette-forming epithelial cells, highlighting the importance of combining quercetin treatment with sufficient in vitro differentiation (e.g., 28 days) (Figure 7).

In addition, we established an efficient in vitro differentiation protocol based on a spotting method, in which a smaller number of initial cells are grown and differentiated using physical separation into spots of high-cell density, resulting in significantly decreased cell loss and in production of healthier mDA cells, with markedly fewer dead or dying cells (Figure 4) compared with traditional confluent monolayers. Importantly, the monolayer culture medium, but not that of the spotting cultures, became significantly more acidic regardless of frequency of media change (Supplemental Figure 6B), probably contributing to poor cell health in the monolayer cultures. When such d28 cells were further differentiated in vitro, they matured and prominently released DA (3.1 ng/ $\mathrm{ml}$ ) on $\mathrm{d} 47$ and exhibited characteristic mDAN electrophysiological properties by $\mathrm{d} 70$. These data show that cultures on $\mathrm{d} 28$ of this differentiation protocol consist mostly of authentic mDAPs and represent a promising source for transplantation. We successfully scaled up this protocol at a GMP facility to produce clinically relevant quantities of high-quality mDAPs (Supplemental Figure 14).

Although numerous studies have demonstrated highly efficient differentiation of hESCs/hiPSCs into the mDA phenotype, their in vivo efficacy has been variable at best and often has correlated poorly with in vitro data (7). Thus, it is essential that the efficacy of the proposed cell grafts should first be confirmed by several criteria using in vivo animal models of PD: (a) sufficient mDANs differentiate and survive long term in the graft; (b) these mDANs extensively reinnervate target areas in the host STR; and (c) motor dysfunction is substantially improved in multiple appropriate behavioral tests. When $\mathrm{d} 28 \mathrm{C} 4$ cells were transplanted into unilaterally 6-OHDA lesioned Taconic or Charles River athymic rats, DA yields were high and grafts displayed extensive and appropriate reinnervation of host structures. Grafting resulted in complete recovery of pharmacologically induced rotation behavior. The DA yield (ratio of surviving DA neurons to number of transplanted cells) and the degree of behavioral recovery in this study were markedly higher than in comparable hiPSC-based studies (Supplemental Table 6) (44, 45, 57-64). Notably, recovery of rotation behavior was sustained up to 52 weeks (Supplemental Figure $6 \mathrm{M}$ ) and significant recovery was observed in several tests, including corridor, cylinder, and stepping tests (Figure 8), which because they are spontaneous and not pharmacologically stimulated, may be closer analogs of clinical PD symptomatology.

To establish the clinical validity of hiPSC-based personalized cell therapy, it is important to compare the therapeutic product to an established gold standard. In the stem cell field, H9 hESCs have represented this standard for human pluripotent stem cells and human fetal VM cells have been the gold standard as a transplantable cell source for PD. Parmar and colleagues elegantly compared the efficacy of H9-derived mDA cells with human fetal VM cells for in vivo efficacy in restoration of motor function, demonstrating H9-derived DA cells to be as effective as human fetal VM cells (53). Our transplantation study confirmed an identical degree and time course of recovery of rotational behavior whether H9 (hESC) or C4 (hiPSC) was the source (Supplemental Figure 12A). By inference, these data strongly suggest that DA cells generated by our protocol from patient-derived hiPSCs are functionally as effective as fetal VM cells. We also note that a recent study by Takahashi et al. elegantly showed that hiPSC-derived DA cells can survive and ameliorate motor behaviors in MPTP-lesioned monkey models (65), but direct comparison of those results with our study is hindered by the different species platform used. We found that fresh and cryopreserved $\mathrm{C} 4 \mathrm{~d} 28$ cells resulted in similar yields of surviving DA neurons and behavioral improvement in all tests, suggesting that mDAPs derived from hiPSCs can be cryopreserved, stored, and shipped to the surgical location for transplantation. The importance of this to developing a practical and cost-effective clinical therapy cannot be overstated.

In sum, our results support the promise of these techniques to provide clinically applicable personalized autologous cell therapy for PD. We recognize that this methodology is likely to be more costly in dollars and labor than techniques using offthe-shelf methods and allogenic cell lines. Nevertheless, the cost for autologous cell therapy may be expected to decrease steadily with technological refinement and automation, as evidenced, for example, by the dramatic decrease of the costs for WGS (66). Given the substantial advantages inherent in a cell source free of ethical concerns and with the potential to obviate the need for immunosuppression, with its attendant costs and dangers, we propose that the scientific and technical progress described here for hiPSC-based autologous cell therapy for PD will contribute to its clinical application.

\section{Methods}

See Supplemental Methods for additional information.

Experimental animals. Strain details and number of animals in each group are as follows: 6-OHDA lesioned athymic rats (NTac:NIHFoxn1 ${ }^{\text {rnu }}$, Taconic Biosciences), males, 12 to 14 weeks old; athymic rats (Crl:NIH-Foxn1 $1^{\text {rnu }}$, Charles River, stain code 316), males, 12 to 14 weeks old; NOD SCID (NOD.CB17-Prkdc sid /NCrCrl, Charles River, stain code 394 ), males or females, 8 to 10 weeks old. All animals were housed in ventilated cages under a 12-hour light/12-hour dark cycle with ad libitum access to sterile food and water. 
Cell culture. Human BJ dermal fibroblasts (hDF) and HEK 293T cells were purchased from ATCC and grown according to previously published protocols (19). For hiPSC induction, infected cells were maintained in induction medium containing DMEM/F-12, $2 \mathrm{mM} \mathrm{L-glu-}$ tamine, 10\% FBS, $1 \times$ NEAA, $1 \mathrm{mM}$ NAM, $25 \mathrm{mM} \mathrm{NaB}$, and $50 \mu \mathrm{g} / \mathrm{ml}$ AA for 5 days after transfection, then maintained in hiPSC medium containing DMEM/F-12, 2 mM L-glutamine, 20\% KSR, 1× NEAA, 1 mM NAM, $25 \mathrm{mM} \mathrm{NaB}$, and $10 \mathrm{ng} / \mathrm{ml}$ bFGF. The H9 hESC line was obtained from WiCell Institute. All hiPSC lines were maintained in Essential 8 Medium (Thermo Fisher Scientific) using Matrigel matrix and passaged using 0.5 mM EDTA solution for gentle dissociation. All hESC lines were maintained in mTeSR 1 medium (STEMCELL Technologies) using Matrigel matrix. No cell lines used in this study are listed in the International Cell Line Authentication Committee (ICLAC) Register of Misidentified Cell Lines (https://iclac.org/databases/crosscontaminations/) or the NCBI Biosample database (https://www.ncbi. nlm.nih.gov/biosample?term $=$ misidentified +cell+line $\% 5$ bFilter $\% 5 \mathrm{~d}$ ) of misidentified cell lines. All cell lines were authenticated by interspecies determination (isoenzyme analysis and STR analysis) by the providing company and routinely tested for mycoplasma detection.

Study approval. Animal studies were performed in accordance with current NIH guidelines and McLean Hospital/Harvard University IACUC protocols (2015N000001 and 2015N000002). Skin biopsies from 3 healthy subjects and 1 sporadic PD patient were taken with written informed consent under an IRB-approved protocol (Partners IRB2010P001100).

\section{Author contributions}

KSK conceived and supervised the project. Co-first authors BS, YC, SK, JJ, NL, and HS designed and performed the experiments; listing order reflects the time contribution of each. KJP and VYB contributed to electrophysiology. IHL and SWK performed the bioinformatics analysis. CL, MF, MJL, JHJ, JK, and DH performed the experiments. BMC, MHT, PL, BSC, JHK, and JSS analyzed the data. KSK, JSS, and BS wrote the paper with input from all coauthors.

\section{Acknowledgments}

We would like to thank all members of the molecular neurobiology laboratory past and present who participated in the project. In particular, we thank Sangmi Chung and Kai Sonntag for technical input and helpful discussions. We also thank the Mostoslavsky's lab for gifting us the STEMCCA lentiviral vector. We would like to acknowledge the personnel of the Dana Farber Cancer Institute Cell Manipulation Core Facility, in particular Jerome Ritz, Sarah Nikiforow, Hélène Negre, and Laeticia Pinte, for their technical and administrative help in setting up the GMP cell production. This work was supported by NIH grants (NS070577, NS084869, and OD024622) and a National Research Foundation (NRF) grant (2017R1A2B4008456 to HS) as well as the Parkinson's Cell Therapy Research Fund at McLean Hospital and Massachusetts General Hospital.

Address correspondence to: Kwang-Soo Kim, Molecular Neurobiology Lab, Room 216, McLean Hospital, 115 Mill Street, Belmont, Massachusetts 02478, USA. Phone: 617.855.2024; Email: kskim@ mclean.harvard.edu. Or to: Jeffrey S. Schweitzer, Department of Neurosurgery, Massachusetts General Hospital, 15 Parkman Street Wang 745, Boston, Massachusetts 02114, USA. Phone: 617.726.1799; Email: jschweitzer1@mgh.harvard.edu.
1. de Lau LM, Breteler MM. Epidemiology of Parkinson's disease. Lancet Neurol. 2006;5(6):525-535.

2. Kang UJ, Fahn S. Management of tardive dyskinesia. Ration Drug Ther. 1988;22(8):1-7.

3. Bjorklund A, Kordower JH. Cell therapy for Parkinson's disease: what next? Mov Disord. 2013;28(1):110-115.

4. Kefalopoulou Z, et al. Long-term clinical outcome of fetal cell transplantation for Parkinson disease: two case reports. JAMA Neurol. 2014;71(1):83-87.

5. Barker RA, Drouin-Ouellet J, Parmar M. Cellbased therapies for Parkinson disease-past insights and future potential. Nat Rev Neurol. 2015;11(9):492-503.

6. Lindvall O. Clinical translation of stem cell transplantation in Parkinson's disease. J Intern Med. 2016;279(1):30-40.

7. Sonntag KC, et al. Pluripotent stem cell-based therapy for Parkinson's disease: Current status and future prospects. Prog Neurobiol. 2018;168:1-20.

8. Takahashi K, Yamanaka S. Induction of pluripotent stem cells from mouse embryonic and adult fibroblast cultures by defined factors. Cell. 2006;126(4):663-676.

9. Takahashi $\mathrm{K}$, et al. Induction of pluripotent stem cells from adult human fibroblasts by defined factors. Cell. 2007;131(5):861-872.

10. Yu J, et al. Induced pluripotent stem cell lines derived from human somatic cells. Science. 2007;318(5858):1917-1920.
11. Park IH, et al. Reprogramming of human somatic cells to pluripotency with defined factors. Nature. 2008;451(7175):141-146.

12. Scudellari M. How iPS cells changed the world. Nature. 2016;534(7607):310-312.

13. Takahashi K, Yamanaka S. A decade of transcription factor-mediated reprogramming to pluripotency. Nat Rev Mol Cell Biol. 2016;17(3):183-193.

14. Tapia N, Schöler HR. Molecular obstacles to clinical translation of iPSCs. Cell Stem Cell. 2016;19(3):298-309.

15. Miura K, et al. Variation in the safety of induced pluripotent stem cell lines. Nat Biotechnol. 2009;27(8):743-745.

16. Ohnishi K, et al. Premature termination of reprogramming in vivo leads to cancer development through altered epigenetic regulation. Cell. 2014;156(4):663-677.

17. Mandai M, et al. Autologous induced stem-cellderived retinal cells for macular degeneration. $N$ Engl J Med. 2017;376(11):1038-1046.

18. Kirkeby A, et al. Predictive markers guide differentiation to improve graft outcome in clinical translation of hESC-based therapy for Parkinson's disease. Cell Stem Cell. 2017;20(1):135-148.

19. Cha Y, et al. Metabolic control of primed human pluripotent stem cell fate and function by the miR-200c-SIRT2 axis. Nat Cell Biol. 2017;19(5):445-456.

20. Korpal M, Lee ES, Hu G, Kang Y. The miR-200 family inhibits epithelial-mesenchymal transition and cancer cell migration by direct targeting of E-cadherin transcriptional repressors ZEB1 and ZEB2. J Biol Chem. 2008;283(22):14910-14914.

21. Valastyan S, Weinberg RA. Roles for microRNAs in the regulation of cell adhesion molecules. $J$ Cell Sci. 2011;124(Pt 7):999-1006.

22. Banyard J, et al. Regulation of epithelial plasticity by miR-424 and miR-200 in a new prostate cancer metastasis model. Sci Rep. 2013;3:3151.

23. Pieters T, van Roy F. Role of cell-cell adhesion complexes in embryonic stem cell biology. J Cell Sci. 2014;127(Pt 12):2603-2613.

24. Chan EM, et al. Live cell imaging distinguishes bona fide human iPS cells from partially reprogrammed cells. Nat Biotechnol. 2009;27(11):1033-1037.

25. Tanabe K, Nakamura M, Narita M, Takahashi K, Yamanaka S. Maturation, not initiation, is the major roadblock during reprogramming toward pluripotency from human fibroblasts. Proc Natl Acad Sci USA. 2013;110(30):12172-12179.

26. Nakagawa M, Takizawa N, Narita M, Ichisaka T, Yamanaka S. Promotion of direct reprogramming by transformation-deficient Myc. Proc Natl Acad Sci USA. 2010;107(32):14152-14157.

27. Kandoth C, et al. Mutational landscape and significance across 12 major cancer types. Nature. 2013;502(7471):333-339.

28. Merkle FT, et al. Human pluripotent stem cells 
recurrently acquire and expand dominant negative P53 mutations. Nature. 2017;545(7653):229-233.

29. Kilpinen $\mathrm{H}$, et al. Common genetic variation drives molecular heterogeneity in human iPSCs. Nature. 2017;546(7658):370-375.

30. Martincorena I, Campbell PJ. Somatic mutation in cancer and normal cells. Science. 2015;349(6255):1483-1489.

31. Chung S, et al. Wnt1-lmx1a forms a novel autoregulatory loop and controls midbrain dopaminergic differentiation synergistically with the SHH-FoxA2 pathway. Cell Stem Cell. 2009;5(6):646-658.

32. Joksimovic M, et al. Spatiotemporally separable Shh domains in the midbrain define distinct dopaminergic progenitor pools. Proc Natl Acad Sci USA. 2009;106(45):19185-19190.

33. Joksimovic M, et al. Wnt antagonism of Shh facilitates midbrain floor plate neurogenesis. Nat Neurosci. 2009;12(2):125-131.

34. Arenas E, Denham M, Villaescusa JC. How to make a midbrain dopaminergic neuron. Development. 2015;142(11):1918-1936.

35. Tao Y, Zhang SC. Neural subtype specification from human pluripotent stem cells. Cell Stem Cell. 2016;19(5):573-586.

36. Chambers SM, Fasano CA, Papapetrou EP, Tomishima M, Sadelain M, Studer L. Highly efficient neural conversion of human ES and iPS cells by dual inhibition of SMAD signaling. Nat Biotechnol. 2009;27(3):275-280.

37. Kriks $S$, et al. Dopamine neurons derived from human ES cells efficiently engraft in animal models of Parkinson's disease. Nature. 2011;480(7378):547-551.

38. Porter AG, Jänicke RU. Emerging roles of caspase-3 in apoptosis. Cell Death Differ. 1999;6(2):99-104.

39. Cummings BS, Schnellmann RG. Measurement of cell death in mammalian cells. Curr Protoc Pharmacol. 2004; Chapter 12:Unit 12.8.

40. Lee MO, et al. Inhibition of pluripotent stem cell-derived teratoma formation by small molecules. Proc Natl Acad Sci USA. 2013;110(35):E3281-E3290.

41. Pennartz S, et al. Purification of neuronal precursors from the adult mouse brain: comprehensive gene expression analysis provides new insights into the control of cell migration, differentiation, and homeostasis. Mol Cell Neurosci. 2004;25(4):692-706.

42. Jiang $\mathrm{Y}$, et al. Essential role for survivin in early brain development. J Neurosci. 2005;25(30):6962-6970.

43. Darband SG, et al. Quercetin: A functional dietary flavonoid with potential chemo-preventive properties in colorectal cancer. JCell Physiol. 2018;233(9):6544-6560.

44. Doi D, et al. Isolation of human induced pluripotent stem cell-derived dopaminergic progenitors by cell sorting for successful transplantation. Stem Cell Reports. 2014;2(3):337-350.

45. Samata B, et al. Purification of functional human ES and iPSC-derived midbrain dopaminergic progenitors using LRTM1. Nat Commun . 2016;7:13097.

46. Guzman JN, Sánchez-Padilla J, Chan CS, Surmeier DJ. Robust pacemaking in substantia nigra dopaminergic neurons. J Neurosci. 2009;29(35):11011-11019.

47. Ungerstedt U, Arbuthnott GW. Quantitative recording of rotational behavior in rats after 6-hydroxy-dopamine lesions of the nigrostriatal dopamine system. Brain Res. 1970;24(3):485-493.

48. Tieu K. A guide to neurotoxic animal models of Parkinson's disease. Cold Spring Harb Perspect Med. 2011;1(1):a009316.

49. Blandini F, Armentero MT. Animal models of Parkinson's disease. FEBS J. 2012;279(7):1156-1166.

50. Dowd E, Monville C, Torres EM, Dunnett SB. The Corridor Task: a simple test of lateralised response selection sensitive to unilateral dopamine deafferentation and graft-derived dopamine replacement in the striatum. Brain Res Bull. 2005;68(1-2):24-30.

51. Olsson M, Nikkhah G, Bentlage C, Bjorklund A. Forelimb akinesia in the rat Parkinson model: differential effects of dopamine agonists and nigral transplants as assessed by a new stepping test. J Neurosci. 1995;15(5 Pt 2):3863-3875.

52. Lundblad M, Andersson M, Winkler C, Kirik D, Wierup N, Cenci MA. Pharmacological validation of behavioural measures of akinesia and dyskinesia in a rat model of Parkinson's disease. Eur J Neurosci. 2002;15(1):120-132.

53. Grealish S, et al. Human ESC-derived dopamine neurons show similar preclinical efficacy and potency to fetal neurons when grafted in a rat model of Parkinson's disease. Cell Stem Cell. 2014;15(5):653-665.

54. Barker RA, Parmar M, Studer L, Takahashi J. Human trials of stem cell-derived dopamine neurons for Parkinson's disease: dawn of a new era. Cell Stem Cell. 2017;21(5):569-573.
55. Ostrom QT, et al. The epidemiology of glioma in adults: a "state of the science" review. Neuro-oncology. 2014;16(7):896-913.

56. Katsukawa M, Nakajima Y, Fukumoto A, Doi D, Takahashi J. Fail-safe therapy by gamma-ray irradiation against tumor formation by human-induced pluripotent stem cell-derived neural progenitors. Stem Cells Dev. 2016;25(11):815-825.

57. Hargus G, et al. Differentiated Parkinson patient-derived induced pluripotent stem cells grow in the adult rodent brain and reduce motor asymmetry in Parkinsonian rats. Proc Natl Acad Sci USA. 2010;107(36):15921-15926.

58. Rhee YH, et al. Protein-based human iPS cells efficiently generate functional dopamine neurons and can treat a rat model of Parkinson disease. JClin Invest. 2011;121(6):2326-2335.

59. Effenberg A, et al. Striatal transplantation of human dopaminergic neurons differentiated from induced pluripotent stem cells derived from umbilical cord blood using lentiviral reprogramming. Cell Transplant. 2015;24(10):2099-2112.

60. Samata B, et al. X-linked severe combined immunodeficiency (X-SCID) rats for xeno-transplantation and behavioral evaluation. JNeurosci Methods. 2015;243:68-77.

61. Kikuchi T, et al. Idiopathic Parkinson's disease patient-derived induced pluripotent stem cells function as midbrain dopaminergic neurons in rodent brains. J Neurosci Res. 2017;95(9):1829-1837.

62. Lehnen D, et al. IAP-based cell sorting results in homogeneous transplantable dopaminergic precursor cells derived from human pluripotent stem cells. Stem Cell Reports. 2017;9(4):1207-1220.

63. Wakeman DR, et al. Cryopreservation maintains functionality of human iPSC dopamine neurons and rescues parkinsonian phenotypes in vivo. Stem Cell Reports. 2017;9(1):149-161.

64. Swistowski A, et al. Efficient generation of functional dopaminergic neurons from human induced pluripotent stem cells under defined conditions. Stem Cells. 2010;28(10):1893-1904.

65. Kikuchi T, et al. Human iPS cell-derived dopaminergic neurons function in a primate Parkinson's disease model. Nature. 2017;548(7669):592-596.

66. National Human Genome Research Institute. The Cost of Sequencing a Human Genome. NIH website. https://www.genome.gov/aboutgenomics/fact-sheets/Sequencing-HumanGenome-cost. Updated October 30, 2019. Accessed December 10, 2020. 